\title{
A MODAL SYNTHESIS METHOD FOR THE ELASTOACOUSTIC VIBRATION PROBLEM
}

\begin{abstract}
Alfredo Bermúdez ${ }^{1}$, Luis Hervella-Nieto ${ }^{2}$ and Rodolfo Rodríguez ${ }^{3}$
Abstract. A modal synthesis method to solve the elastoacoustic vibration problem is analyzed. A two-dimensional coupled fluid-solid system is considered; the solid is described by displacement variables, whereas displacement potential is used for the fluid. A particular modal synthesis leading to a symmetric eigenvalue problem is introduced. Finite element discretizations with Lagrangian elements are considered for solving the uncoupled problems. Convergence for eigenvalues and eigenfunctions is proved, error estimates are given, and numerical experiments exhibiting the good performance of the method are reported.
\end{abstract}

Mathematics Subject Classification. 65N15, 65N25, 74F10.

Received: November 23, 2001.

\section{INTRODUCTION}

The need of computing fluid-solid interactions arises in many important engineering problems. A general overview on the subject can be found in [15], where numerical methods and further references are also given. This paper deals with one of these interactions: the elastoacoustic vibration problem. It concerns the determination of harmonic vibrations of a linear elastic structure interacting with an acoustic (i.e., inviscid, barotropic) fluid. We will approximate the solutions of this problem using a modal synthesis method.

Let us suppose that we want to approximate the solution of a problem defined on a given domain. The modal synthesis method consists of dividing this domain in several subdomains and calculating the lowest frequency eigenfunctions of the spectral problems associated with the restrictions of the original problem to each subdomain. In some modal synthesis methods, a finite number of functions related to the interfaces between each pair of neighboring subdomains must be calculated too. Then, the solution of the original problem is approximated as a linear combination of all these functions.

\footnotetext{
Keywords and phrases. Fluid-structure interaction, elastoacoustic, modal synthesis.

1 Departamento de Matemática Aplicada, Universidade de Santiago de Compostela, 15706 Santiago de Compostela, Spain. e-mail: mabermud@usc.es. Partially supported by research project PGIDT00PXI20701PR. Xunta de Galicia (Spain).

2 Departamento de Matemáticas, Universidade da Coruña, 15071 A Coruña, Spain. Partially supported by FONDAP in Applied Mathematics, Chile.

3 Departamento de Ingeniería Matemática, Universidad de Concepción, Casilla 160-C, Concepción, Chile. Partially supported by FONDECYT Grant 1.990.346 and FONDAP in Applied Mathematics, Chile. 
The main advantage of this technique is that, instead of solving a complex problem, we first solve several simpler problems and, then, a finite dimensional problem on the whole domain, usually with low dimension and good numerical properties.

These methods have been introduced in the context of dynamical analysis of structures by Hurty in [12] and improved by Craig and Bampton in [7]. Further, a modal synthesis method without interface associated functions has been proposed by Goldman in [8].

For the application of these methods, we must have into account that the solutions of the problems on each subdomain are not exactly known in most cases. Then, they must be approximated somehow too (for instance, using a finite element technique).

On the other hand, although the modal synthesis methods are very much used in practical computations, they do not appear frequently in the mathematical bibliography. A good introduction to their analysis can be found in [3], where some modal synthesis methods are studied for a 1D-problem. In this reference the functions of the uncoupled problems are supposed to be exactly known. In [4], the analysis is extended to the $n$-dimensional Laplace problem having into account a finite element discretization.

Other advantage of the component mode synthesis methods is that they allow for a good treatment of problems involving two media with different physical features. This is one reason of their importance in fluidstructure interaction problems, where they are very frequently used (see, for instance $[5,16,18]$ ).

In [15], a modal synthesis method is introduced to solve the elastoacoustic problem, using the non-symmetric potential/displacement formulation. The solutions are approximated by a linear combination of the lowestfrequency eigenfunctions of the fluid in a rigid cavity, the lowest-frequency eigenfunctions of the solid in vacuo, and the static responses of the fluid to the solid eigenfunctions (i.e., the solutions of the static Neumann problem in the fluid with prescribed normal displacements on the boundary induced by the solid eigenfunctions). Then, the test functions are chosen in a different space not including the static responses. The resulting coupled problem is symmetric, low-dimensional, and with good numerical properties.

In this paper we present a mathematical analysis of this method combined with finite element discretizations based on piecewise linear continuous functions in both the solid and the fluid. The resulting uncoupled problems are classical and easy to solve numerically. We restrict our presentation to two-dimensional domains, for technical reasons, but the techniques can be used in 3D situations.

The outline of the paper is as follows: we introduce the potential/displacement formulation for the elastoacoustic problem and characterize its spectrum in Section 2. In Section 3, we introduce the spectral uncoupled problems in fluid and solid and their discretization in the corresponding finite element spaces. In Section 4 we introduce the static lifting in the fluid and its discretization. In Section 5 we define the approximate coupled problem with modal synthesis. In Section 6, we prove several intermediate theoretical results that we use for the analysis of this method in the abstract framework of [13]. In Section 7 we prove the convergence for eigenfunctions and eigenvalues and obtain error estimates. Finally, in Section 8, we report a numerical test that illustrates the good performance of the method.

\section{Statement of the PRoblem}

We consider the problem of determining the small-amplitude coupled motions of an inviscid barotropic fluid contained into a linear elastic structure.

Throughout this paper we use the standard notation for Sobolev spaces. We use boldface to represent linear spaces of vector fields.

Let $\Omega_{\mathrm{F}}$ and $\Omega_{\mathrm{S}}$ be the domains occupied by fluid and solid, respectively, as in Figure 1 . We suppose both are polygonal domains. Let us denote by $\Gamma_{\mathrm{I}}$ the interface between solid and fluid and by $\vec{\nu}$ its unit normal vector pointing outwards $\Omega_{\mathrm{F}}$. We assume that the exterior boundary of the solid is the union of two parts, $\Gamma_{\mathrm{D}}$ and $\Gamma_{\mathrm{N}}$, and that the structure is fixed on $\Gamma_{\mathrm{D}}$ and free of stress on $\Gamma_{\mathrm{N}}$. Finally let $\vec{\eta}$ be the unit outward normal vector along $\Gamma_{\mathrm{N}}$. 


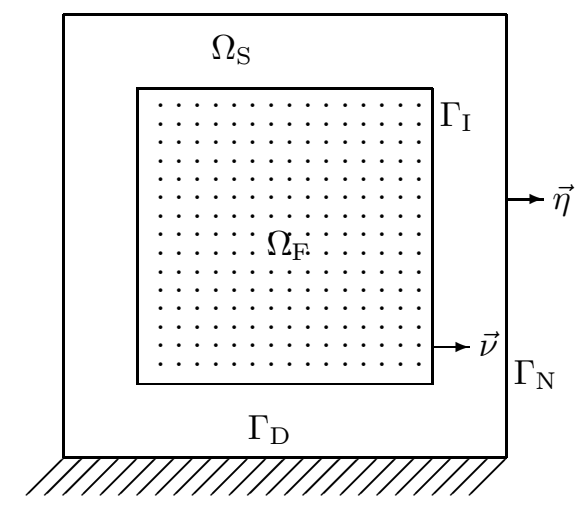

Figure 1. Fluid and solid domains.

The governing equations for free harmonic small amplitude motions of the coupled system are (see, for instance, [15]),

$$
\left\{\begin{array}{rlrl}
\vec{\nabla} p-\omega^{2} \rho_{\mathrm{F}} \vec{u}^{\mathrm{F}} & =\overrightarrow{0} & & \text { in } \Omega_{\mathrm{F}}, \\
p+\rho_{\mathrm{F}} c^{2} \operatorname{div} \vec{u}^{\mathrm{F}} & =0 & & \text { in } \Omega_{\mathrm{F}}, \\
\operatorname{div}[\sigma(\vec{u})]+\omega^{2} \rho_{\mathrm{S}} \vec{u} & =\overrightarrow{0} & & \text { in } \Omega, \\
\vec{u}^{\mathrm{F}} \cdot \vec{\nu} & =\vec{u} \cdot \vec{\nu} & \text { on } \Gamma_{\mathrm{I}}, \\
\sigma(\vec{u}) \vec{\nu} & =-p \vec{\nu} & & \text { on } \Gamma_{\mathrm{I}}, \\
\sigma(\vec{u}) \vec{\eta} & =\overrightarrow{0} & & \text { on } \Gamma_{\mathrm{N}}, \\
\vec{u} & =\overrightarrow{0} & & \text { on } \Gamma_{\mathrm{D}},
\end{array}\right.
$$

where $\omega$ is the frequency of the harmonic motion, $p$ is the fluid pressure and $c$ its acoustic velocity; $\rho_{\mathrm{F}}, \vec{u}^{\mathrm{F}}$ and $\rho_{\mathrm{S}}, \vec{u}$ are the densities and displacements in the fluid and the solid, respectively; $\sigma$ is the stress tensor which is related to $\vec{u}$ by Hooke's law:

$$
\sigma_{i j}(\vec{u})=\lambda_{S} \sum_{k=1}^{2} \epsilon_{k k}(\vec{u}) \delta_{i j}+2 \mu_{S} \epsilon_{i j}(\vec{u}), \quad i, j=1,2
$$

In the previous equation, $\lambda_{S}$ and $\mu_{S}$ denote the Lamé coefficients of the solid and $\epsilon_{i j}(\vec{u})$ the components of the infinitesimal strain tensor given by

$$
\epsilon_{i j}(\vec{u})=\frac{1}{2}\left(\frac{\partial u_{i}}{\partial x_{j}}+\frac{\partial u_{j}}{\partial x_{i}}\right), \quad i, j=1,2 .
$$

According to [15], if we assume $\vec{u}^{\mathrm{F}}=\vec{\nabla} \varphi$, with $\int_{\Omega_{\mathrm{F}}} \varphi \mathrm{d} x=0$, we have

$$
p=\rho_{\mathrm{F}} \omega^{2} \varphi-\frac{\rho_{\mathrm{F}} c^{2}}{\left|\Omega_{\mathrm{F}}\right|} \int_{\Gamma_{\mathrm{I}}} \vec{u}^{\mathrm{F}} \cdot \vec{\nu} \mathrm{d} \Gamma
$$

simply by using the first two equations in (2.1). 
Then we can eliminate $p$ and $\vec{u}^{\mathrm{F}}$ in (2.1) to obtain the following potential/displacement formulation,

$$
\left\{\begin{aligned}
-\rho_{\mathrm{F}} \Delta \varphi+\frac{\rho_{\mathrm{F}}}{\left|\Omega_{\mathrm{F}}\right|} \int_{\Gamma_{\mathrm{I}}} \vec{u} \cdot \vec{\nu} \mathrm{d} \Gamma & =\omega^{2} \frac{\rho_{\mathrm{F}}}{c^{2}} \varphi & & \text { in } \Omega_{\mathrm{F}}, \\
-\operatorname{div}[\sigma(\vec{u})] & =\omega^{2} \rho_{\mathrm{S}} \vec{u} & & \text { in } \Omega_{\mathrm{S}}, \\
\vec{u} \cdot \vec{\nu} & =\frac{\partial \varphi}{\partial \nu} & & \text { on } \Gamma_{\mathrm{I}}, \\
\sigma(\vec{u}) \vec{\nu} & =\left(-\rho_{\mathrm{F}} \omega^{2} \varphi+\frac{\rho_{\mathrm{F}} c^{2}}{\left|\Omega_{\mathrm{F}}\right|} \int_{\Gamma_{\mathrm{I}}} \vec{u} \cdot \vec{\nu} \mathrm{d} \Gamma\right) \vec{\nu} & & \text { on } \Gamma_{\mathrm{I}}, \\
\vec{u} & =\overrightarrow{0} & & \text { on } \Gamma_{\mathrm{D}}, \\
\sigma(\vec{u}) \vec{\eta} & =\overrightarrow{0} & & \text { on } \Gamma_{\mathrm{N}} .
\end{aligned}\right.
$$

We emphasize the coupling condition

$$
\vec{u} \cdot \vec{\nu}=\frac{\partial \varphi}{\partial \nu} \quad \text { on } \Gamma_{\mathrm{I}},
$$

since it plays an important role in the definition of the modal synthesis spaces.

If we denote $\lambda=\omega^{2}$, by multiplying the first two equations in (2.2) by adequate test functions and integrating by parts, it is straightforward to see that if $\lambda$ and $(\varphi, \vec{u}) \neq(0, \overrightarrow{0})$ is a solution of (2.2) then it is also a solution of the following variational spectral problem.

VP: Find a real number $\lambda \in \mathbb{R}$ and $(0, \overrightarrow{0}) \neq(\varphi, \vec{u}) \in \mathbf{V}$, such that

$$
a((\varphi, \vec{u}),(\psi, \vec{v}))=\lambda b((\varphi, \vec{u}),(\psi, \vec{v})) \quad \forall(\psi, \vec{v}) \in \mathbf{V},
$$

where

$$
\begin{aligned}
& a((\varphi, \vec{u}),(\psi, \vec{v})):=\int_{\Omega_{\mathrm{F}}} \rho_{\mathrm{F}} \vec{\nabla} \varphi \cdot \vec{\nabla} \psi \mathrm{d} x+\int_{\Omega_{\mathrm{S}}} \sigma(\vec{u}): \epsilon(\vec{v}) \mathrm{d} x-\int_{\Gamma_{\mathrm{I}}} \rho_{\mathrm{F}} \psi \vec{u} \cdot \vec{\nu} \mathrm{d} \Gamma+\frac{\rho_{\mathrm{F}} c^{2}}{\left|\Omega_{\mathrm{F}}\right|} \int_{\Gamma_{\mathrm{I}}} \vec{u} \cdot \vec{\nu} \mathrm{d} \Gamma \int_{\Gamma_{\mathrm{I}}} \vec{v} \cdot \vec{\nu} \mathrm{d} \Gamma, \\
& b((\varphi, \vec{u}),(\psi, \vec{v})):=\int_{\Omega_{\mathrm{F}}} \frac{\rho_{\mathrm{F}}}{c^{2}} \varphi \psi \mathrm{d} x+\int_{\Omega_{\mathrm{S}}} \rho_{\mathrm{S}} \vec{u} \cdot \vec{v} \mathrm{~d} x+\int_{\Gamma_{\mathrm{I}}} \rho_{\mathrm{F}} \varphi \vec{v} \cdot \vec{\nu} \mathrm{d} \Gamma,
\end{aligned}
$$

and

$$
\mathbf{V}:=\stackrel{\circ}{\mathrm{H}}^{1}\left(\Omega_{\mathrm{F}}\right) \times \mathbf{H}_{\Gamma_{\mathrm{D}}}^{1}\left(\Omega_{\mathrm{S}}\right),
$$

with $\stackrel{\circ}{H}^{1}\left(\Omega_{\mathrm{F}}\right)$ being the set of functions $\psi$ in $\mathrm{H}^{1}\left(\Omega_{\mathrm{F}}\right)$ with $\int_{\Omega_{\mathrm{F}}} \psi \mathrm{d} x=0$ and $\mathbf{H}_{\Gamma_{\mathrm{D}}}^{1}\left(\Omega_{\mathrm{S}}\right)$ the set of functions in $\mathbf{H}^{1}\left(\Omega_{\mathrm{S}}\right)$ with null trace on $\Gamma_{\mathrm{D}}$. We will use the following norms

$$
\begin{aligned}
& \|\psi\|_{\mathrm{F}}^{2}=\int_{\Omega_{\mathrm{F}}} \rho_{\mathrm{F}}|\vec{\nabla} \psi|^{2} \mathrm{~d} x \quad \text { for } \psi \in \stackrel{\circ}{\mathrm{H}}^{1}\left(\Omega_{\mathrm{F}}\right), \\
& \|\vec{v}\|_{\mathrm{S}}^{2}=\int_{\Omega_{\mathrm{S}}} \sigma(\vec{v}): \epsilon(\vec{v}) \mathrm{d} x \quad \text { for } \vec{v} \in \mathbf{H}_{\Gamma_{\mathrm{D}}}^{1}\left(\Omega_{\mathrm{S}}\right),
\end{aligned}
$$

which are equivalent to the standard ones (see [17]). We will use the product norm in $\mathbf{V}$

$$
\|(\psi, \vec{v})\|_{\mathrm{V}}^{2}=\|\psi\|_{\mathrm{F}}^{2}+\|\vec{v}\|_{\mathrm{S}}^{2} .
$$

We notice that, since $a$ and $b$ are not symmetric, the eigenvalues of $\mathbf{V P}$ could be, in principle, complex numbers. However, we have the following: 
Theorem 2.1. The set of eigenvalues of $\mathbf{V P}$ consists of a sequence of positive real numbers converging to $+\infty$. All of them have finite multiplicity and their ascent is one.

Furthermore, there exist constants $r>\frac{1}{2}$ and $s>0$, depending only on the domains $\Omega_{\mathrm{F}}$ and $\Omega_{\mathrm{S}}$ and on the physical parameters, such that any eigenfunction of $\mathbf{V P},(\varphi, \vec{u})$, satisfies

$$
(\varphi, \vec{u}) \in \mathrm{H}^{1+r}\left(\Omega_{\mathrm{F}}\right) \times \mathrm{H}^{1+s}\left(\Omega_{\mathrm{S}}\right)
$$

Proof. It is easy to prove (see [11]) that the potential/displacement formulation of the elastoacoustic problem is the adjoint of the pressure/displacement formulation of the same problem. Then the eigenvalues and their ascents coincide for both problems. Thus the results in [19, Sect. 8], apply to prove the characterization of the spectrum.

We can prove the additional regularity by reasoning as in [2, Lem. 6.2].

\section{UNCOUPLED SPECTRAL PROBLEMS}

We will use two different finite dimensional spaces to approximate $\mathbf{V}$. In order to define these spaces, we need the lowest frequency eigenfunctions of two uncoupled spectral problems in the fluid and in the solid, respectively. In this section we introduce these problems and a finite element discretization to approximate their solutions.

We consider the following spectral problem associated with the Laplacian operator in the fluid domain with homogeneous Neumann boundary conditions.

$\mathbf{V P}^{\mathrm{F}}$ : Find $\lambda^{\mathrm{F}} \in \mathbb{R}$ and $0 \neq \varphi \in \stackrel{\circ}{\mathrm{H}}^{1}\left(\Omega_{\mathrm{F}}\right)$, such that

$$
\int_{\Omega_{\mathrm{F}}} \rho_{\mathrm{F}} \vec{\nabla} \varphi \cdot \vec{\nabla} \psi \mathrm{d} x=\lambda^{\mathrm{F}} \int_{\Omega_{\mathrm{F}}} \frac{\rho_{\mathrm{F}}}{c^{2}} \varphi \psi \mathrm{d} x \quad \forall \psi \in \stackrel{\circ}{\mathrm{H}}^{1}\left(\Omega_{\mathrm{F}}\right) .
$$

The eigenvalues of $\mathbf{V} \mathbf{P}^{\mathrm{F}}$ form an increasing sequence of real numbers going to infinity. We denote $\left(\lambda_{i}^{\mathrm{F}}, \varphi_{i}\right)_{i \geq 1}$ the solutions of $\mathbf{V} \mathbf{P}^{\mathrm{F}}$, where the eigenvalues are repeated according to their multiplicities.

We consider the spectral problem associated with the linear elasticity operator in the solid domain with homogeneous Dirichlet conditions on $\Gamma_{\mathrm{D}}$ and homogeneous Neumann conditions on $\Gamma_{\mathrm{N}}$, namely,

$\mathbf{V P}^{\mathrm{S}}$ : Find $\lambda^{\mathrm{S}} \in \mathbb{R}$ and $\overrightarrow{0} \neq \vec{u} \in \mathbf{H}_{\Gamma_{\mathrm{D}}}^{1}\left(\Omega_{\mathrm{S}}\right)$, such that

$$
\int_{\Omega_{\mathrm{S}}} \sigma(\vec{u}): \epsilon(\vec{v}) \mathrm{d} x=\lambda^{\mathrm{S}} \int_{\Omega_{\mathrm{S}}} \rho_{\mathrm{S}} \vec{u} \cdot \vec{v} \mathrm{~d} x \quad \forall \vec{v} \in \mathbf{H}_{\Gamma_{\mathrm{D}}}^{1}\left(\Omega_{\mathrm{S}}\right) .
$$

We denote the solutions of this problem by $\left(\lambda_{m}^{\mathrm{S}}, \vec{u}_{m}\right)_{m \geq 1}$, where the eigenvalues are repeated according to their multiplicities.

We have the following a priori estimate for the solutions of these problems:

Lemma 3.1. Let $r$ and $s$ be the constants in Theorem 2.1. Then we have

- $\varphi_{i} \in \mathrm{H}^{1+r}\left(\Omega_{\mathrm{F}}\right), \forall i \geq 1$ and $\left\|\varphi_{i}\right\|_{1+r, \Omega_{\mathrm{F}}} \leq C \lambda_{i}^{\mathrm{F}}\left\|\varphi_{i}\right\|_{0, \Omega_{\mathrm{F}}}$,

- $\vec{u}_{m} \in \mathbf{H}^{1+s}\left(\Omega_{\mathrm{S}}\right), \forall m \geq 1$ and $\left\|\vec{u}_{m}\right\|_{1+s, \Omega_{\mathrm{S}}} \leq C \lambda_{m}^{\mathrm{S}}\left\|\vec{u}_{m}\right\|_{0, \Omega_{\mathrm{S}}}$,

where constants are independent of $i$ and $m$, respectively.

Proof. It is a direct consequence of the usual a priori estimates for the Poisson's problem and for the linear elasticity problem in a polygon (see [9]) and of the continuity of their solutions with respect to the right-hand side. 
We choose the lowest frequency eigenfunctions in each uncoupled problem and define the space

$$
\mathbf{V}^{N, 1}=\left\langle\left\{\left(\varphi_{i}, \overrightarrow{0}\right)\right\}_{i=1}^{N_{\mathrm{F}}} \cup\left\{\left(0, \vec{u}_{m}\right)\right\}_{m=1}^{N_{\mathrm{S}}}\right\rangle,
$$

where $N=\left(N_{\mathrm{F}}, N_{\mathrm{S}}\right)$.

Notice that any pair $(\varphi, \vec{u}) \in \mathbf{V}^{N, 1}$ satisfies $\left.\frac{\partial \varphi}{\partial \nu}\right|_{\Gamma_{\mathrm{I}}}=0$, but, in general, $\vec{u} \cdot \nu \neq 0$ on $\Gamma_{\mathrm{I}}$. Thus, the functions in $\mathbf{V}^{N, 1}$ do not satisfy the coupling condition (2.3).

We need to approximate the functions in $\mathbf{V}^{N, 1}$. For so doing we introduce finite element discretizations of problems $\mathbf{V P} \mathbf{P}^{F}$ and $\mathbf{V P} \mathbf{P}^{S}$.

Let $\left\{\mathcal{T}_{h}^{\mathrm{F}}\right\}$ and $\left\{\mathcal{T}_{h}^{\mathrm{S}}\right\}$ be two families of regular triangulations of $\Omega_{\mathrm{F}}$ and $\Omega_{\mathrm{S}}$, respectively. We assume, for simplicity, that for each $h$ the triangulations $\mathcal{T}_{h}^{\mathrm{F}}$ and $\mathcal{T}_{h}^{\mathrm{S}}$ are compatible on the contact interface $\Gamma_{\mathrm{I}}$.

Let

$$
\begin{aligned}
& \mathrm{L}_{h}\left(\Omega_{\mathrm{F}}\right):=\left\{\psi_{h} \in \mathrm{H}^{1}\left(\Omega_{\mathrm{F}}\right):\left.\psi_{h}\right|_{T} \in \mathcal{P}_{1}(T), \quad \forall T \in \mathcal{T}_{h}^{\mathrm{F}}\right\}, \\
& \mathrm{L}_{h}\left(\Omega_{\mathrm{S}}\right):=\left\{v_{h} \in \mathrm{H}^{1}\left(\Omega_{\mathrm{S}}\right):\left.v_{h}\right|_{T} \in \mathcal{P}_{1}(T), \quad \forall T \in \mathcal{T}_{h}^{\mathrm{S}}\right\} .
\end{aligned}
$$

We use the following discrete spaces for fluid and solid,

$$
\begin{aligned}
& \mathrm{V}_{h}^{\mathrm{F}}:=\left\{\psi_{h} \in \mathrm{L}_{h}\left(\Omega_{\mathrm{F}}\right): \int_{\Omega_{\mathrm{F}}} \psi_{h} \mathrm{~d} x=0\right\}, \\
& \mathbf{V}_{h}^{\mathrm{S}}:=\left\{\vec{v}_{h} \in \mathrm{L}_{h}\left(\Omega_{\mathrm{S}}\right)^{2}:\left.\quad \vec{v}_{h}\right|_{\Gamma_{\mathrm{D}}}=\overrightarrow{0}\right\} .
\end{aligned}
$$

Then the approximate uncoupled spectral problem in the fluid is

$\mathbf{V P}_{h}^{\mathrm{F}}$ : Find $\lambda_{h}^{\mathrm{F}} \in \mathbb{R}, 0 \neq \varphi_{h} \in \mathrm{V}_{h}^{\mathrm{F}}$, such that

$$
\int_{\Omega_{\mathrm{F}}} \rho_{\mathrm{F}} \vec{\nabla} \varphi_{h} \cdot \vec{\nabla} \psi_{h} \mathrm{~d} x=\lambda_{h}^{\mathrm{F}} \int_{\Omega_{\mathrm{F}}} \frac{\rho_{\mathrm{F}}}{c^{2}} \varphi_{h} \psi_{h} \mathrm{~d} x \quad \forall \psi_{h} \in \mathrm{V}_{h}^{\mathrm{F}}
$$

Let us remark that it is not necessary to impose the zero-mean condition. More precisely, $\mathbf{V P}_{h}^{\mathrm{F}}$ is equivalent to solve:

Find $\lambda_{h}^{\mathrm{F}} \in \mathbb{R}, \lambda_{h}^{\mathrm{F}} \neq 0, \varphi_{h} \in \mathrm{L}_{h}\left(\Omega_{\mathrm{F}}\right), \varphi_{h} \neq 0$, such that

$$
\int_{\Omega_{\mathrm{F}}} \rho_{\mathrm{F}} \vec{\nabla} \varphi_{h} \cdot \vec{\nabla} \psi_{h} \mathrm{~d} x=\lambda_{h}^{\mathrm{F}} \int_{\Omega_{\mathrm{F}}} \frac{\rho_{\mathrm{F}}}{c^{2}} \varphi_{h} \psi_{h} \mathrm{~d} x \quad \forall \psi_{h} \in \mathrm{L}_{h}\left(\Omega_{\mathrm{F}}\right)
$$

Indeed, if $\left(\lambda_{h}^{\mathrm{F}}, \varphi_{h}\right)$ is solution of (3.1) then $\varphi_{h}$ must be orthogonal to the constant functions, since these functions constitute the eigenspace associated to the eigenvalue 0.

Let $\tilde{N}_{\mathrm{F}}^{h}$ be the number of degrees of freedom of $\mathrm{V}_{h}^{\mathrm{F}}$. We denote the discrete eigenpairs of $\mathbf{V P}_{h}^{\mathrm{F}}$ by $\left(\lambda_{i h}^{\mathrm{F}}, \varphi_{i h}\right)_{i=1}^{\tilde{N}_{\mathrm{F}}^{h}}$. We define the generalized mass of each eigenfunction as

$$
\mu_{i h}^{\mathrm{F}}=\int_{\Omega_{\mathrm{F}}} \rho_{\mathrm{F}}\left|\vec{\nabla} \varphi_{i h}\right|^{2} \mathrm{~d} x .
$$

Then the following orthogonality properties are verified,

$$
\begin{aligned}
\int_{\Omega_{\mathrm{F}}} \rho_{\mathrm{F}} \vec{\nabla} \varphi_{i h} \cdot \vec{\nabla} \varphi_{j h} \mathrm{~d} x & =\delta_{i j} \mu_{i h}^{\mathrm{F}}, \\
\int_{\Omega_{\mathrm{F}}} \frac{\rho_{\mathrm{F}}}{c^{2}} \varphi_{i h} \varphi_{j h} \mathrm{~d} x & =\delta_{i j} \frac{\mu_{i h}^{\mathrm{F}}}{\lambda_{i h}^{\mathrm{F}}} .
\end{aligned}
$$


Now we define the approximate uncoupled spectral problem in the solid:

$\mathbf{V P}_{h}^{\mathrm{S}}$ : Find $\lambda_{h}^{\mathrm{S}} \in \mathbb{R}, \overrightarrow{0} \neq \vec{u}_{h} \in \mathbf{V}_{h}^{\mathrm{S}}$, such that

$$
\int_{\Omega_{\mathrm{S}}} \sigma\left(\vec{u}_{h}\right): \epsilon\left(\vec{v}_{h}\right) \mathrm{d} x=\lambda_{h}^{\mathrm{S}} \int_{\Omega_{\mathrm{S}}} \rho_{\mathrm{S}} \vec{u}_{h} \cdot \vec{v}_{h} \mathrm{~d} x \quad \forall \vec{v}_{h} \in \mathbf{V}_{h}^{\mathrm{S}} .
$$

Let $\tilde{N}_{\mathrm{S}}^{h}$ be the number of degrees of freedom of $\mathbf{V}_{h}^{\mathrm{S}}$. We denote the solutions of this problem $\left(\lambda_{m h}^{\mathrm{S}}, \vec{u}_{m h}\right)_{m=1}^{\tilde{N}_{\mathrm{S}}^{h}}$. We define the generalized mass for the solid eigenfunctions

$$
\mu_{m h}^{\mathrm{S}}=\int_{\Omega_{\mathrm{S}}} \rho_{\mathrm{S}}\left|\vec{u}_{m h}\right|^{2} \mathrm{~d} x
$$

Then

$$
\begin{gathered}
\int_{\Omega_{\mathrm{S}}} \sigma\left(\vec{u}_{m h}\right): \epsilon\left(\vec{u}_{n h}\right) \mathrm{d} x=\delta_{m n} \lambda_{m h}^{\mathrm{S}} \mu_{m h}^{\mathrm{S}} \\
\int_{\Omega_{\mathrm{S}}} \rho_{\mathrm{S}} \vec{u}_{m h} \cdot \vec{u}_{n h} \mathrm{~d} x=\delta_{m n} \mu_{m h}^{\mathrm{S}} .
\end{gathered}
$$

We have the following estimates for the distance between the solutions of the continuous and the discrete uncoupled spectral problems.

Lemma 3.2. Let $r$ and $s$ be the constants in Theorem 2.1. Then there exist constants $C$ and $h_{0}$ such that the eigenvectors $\varphi_{i}, i \geq 1$ and $\vec{u}_{m}, m \geq 1$ can be chosen so that, for $h \leq h_{0}$,

(i) $\left\|\varphi_{i}-\varphi_{i h}\right\|_{\mathrm{F}} \leq C h^{r}\left\|\varphi_{i}\right\|_{1+r, \Omega_{\mathrm{F}}}$

(ii) $\left\|\vec{u}_{m}-\vec{u}_{m h}\right\|_{\mathrm{S}} \leq C h^{s}\left\|\vec{u}_{m}\right\|_{1+s, \Omega_{\mathrm{S}}}$.

Proof. It is a direct consequence of Theorem 9.1 in [1].

Finally, we consider the $N_{\mathrm{F}}$ lowest frequency eigenmodes of the fluid and the $N_{\mathrm{S}}$ lowest frequency eigenmodes of the solid, with $N_{\mathrm{F}} \leq \tilde{N}_{\mathrm{F}}^{h}$ and $N_{\mathrm{S}} \leq \tilde{N}_{\mathrm{S}}^{h}$, and define the finite dimensional space

$$
\mathbf{V}_{h}^{N, 1}=\left\langle\left\{\left(\varphi_{i h}, \overrightarrow{0}\right)\right\}_{i=1}^{N_{\mathrm{F}}} \cup\left\{\left(0, \vec{u}_{m h}\right)\right\}_{m=1}^{N_{\mathrm{S}}}\right\rangle
$$

\section{Static Liftings}

We have already remarked that the functions in $\mathbf{V}^{N, 1}$ (analogously, the functions in $\mathbf{V}_{h}^{N, 1}$ ) do not satisfy the coupling condition (2.3). Then, $\mathbf{V}_{h}^{N, 1}$ is not a good space to approximate the solutions of VP. To complete this space we define the static lifting operator. Let us consider the problem

SL: Given a function $\vec{u} \in \mathbf{H}_{\Gamma_{\mathrm{D}}}^{1}\left(\Omega_{\mathrm{S}}\right)$, find $\varphi^{\vec{u} \cdot \vec{\nu}} \in \mathrm{H}^{1}\left(\Omega_{\mathrm{F}}\right)$ as the only function in $\stackrel{\circ}{H}^{1}\left(\Omega_{\mathrm{F}}\right)$ such that

$$
\int_{\Omega_{\mathrm{F}}} \vec{\nabla} \varphi^{\vec{u} \cdot \vec{\nu}} \cdot \vec{\nabla} \psi \mathrm{d} x=\int_{\Gamma_{\mathrm{I}}} \psi \vec{u} \cdot \vec{\nu} \mathrm{d} \Gamma \quad \forall \psi \in \stackrel{\circ}{\mathrm{H}}^{1}\left(\Omega_{\mathrm{F}}\right) .
$$

We notice that $\frac{\partial \varphi^{\vec{u} \cdot \vec{\nu}}}{\partial \nu}=\vec{u} \cdot \vec{\nu}$ on $\Gamma_{\mathrm{I}}$. Function $\varphi^{\vec{u} \cdot \vec{\nu}}$ will be called static lifting of $\vec{u} \cdot \vec{\nu}$.

We will use the static liftings of the solid eigenfunctions. In order to simplify the notation we write $\varphi^{m} \equiv$ $\varphi^{\vec{u}_{m} \cdot \vec{\nu}}$. 
Let

$$
\mathbf{V}^{N, 2}=\left\langle\left\{\left(\varphi_{i}, \overrightarrow{0}\right)\right\}_{i=1}^{N_{\mathrm{F}}} \cup\left\{\left(\varphi^{m}, \vec{u}_{m}\right)\right\}_{m=1}^{N_{\mathrm{S}}}\right\rangle
$$

Clearly, any $(\varphi, \vec{u}) \in \mathbf{V}^{N, 2}$ satisfies condition (2.3).

We now introduce the discrete static lifting operator associated with the discrete solid eigenfunctions.

$\mathbf{S L}_{h}:$ For $1 \leq m \leq \tilde{N}_{\mathrm{S}}^{h}$, let $\varphi_{h}^{m} \in \mathrm{V}_{h}^{\mathrm{F}}$ be the solution of

$$
\int_{\Omega_{\mathrm{F}}} \vec{\nabla} \varphi_{h}^{m} \cdot \vec{\nabla} \psi_{h} \mathrm{~d} x=\int_{\Gamma_{\mathrm{I}}} \psi_{h} \vec{u}_{m h} \cdot \vec{\nu} \mathrm{d} \Gamma \quad \forall \psi_{h} \in \mathrm{V}_{h}^{\mathrm{F}},
$$

where $\mathrm{V}_{h}^{\mathrm{F}}$ is the finite element space introduced in Section 3.

In the following lemma we prove an a priori estimate for the solutions of SL and an error estimate for the distance between $\varphi^{m}$ and $\varphi_{h}^{m}$ :

Lemma 4.1. Let $r$ and $s$ be the constants in Theorem 2.1 and $t=\min \{r, s\}$. There exists $C$, not depending on $m$, such that

(i) $\varphi^{m} \in \mathrm{H}^{1+r}\left(\Omega_{\mathrm{F}}\right), \forall m \geq 1$, and $\left\|\varphi^{m}\right\|_{1+r, \Omega_{\mathrm{F}}} \leq C\left\|\vec{u}_{m}\right\|_{1, \Omega_{\mathrm{S}}}$,

(ii) $\left\|\varphi^{m}-\varphi_{h}^{m}\right\|_{\mathrm{F}} \leq C h^{t}\left\|\vec{u}_{m}\right\|_{1+s, \Omega_{\mathrm{S}}}$.

Proof. (i) is a consequence of the standard a priori estimate for the Laplace's equation and of the fact that $\vec{u}_{m} \cdot \vec{\nu} \in \mathrm{H}^{\frac{1}{2}}\left(\Gamma_{j}\right)$, for any edge $\Gamma_{j}$ of $\Gamma_{\mathrm{I}}$.

To prove (ii) we apply Strang Lemma (see, for instance, [6]):

$$
\left\|\varphi^{m}-\varphi_{h}^{m}\right\|_{\mathrm{F}} \leq C\left[\inf _{\psi_{h} \in \mathrm{V}_{h}^{\mathrm{F}}}\left\|\varphi^{m}-\psi_{h}\right\|_{\mathrm{F}}+\sup _{\psi_{h} \in \mathrm{V}_{h}^{\mathrm{F}}} \frac{\left|\int_{\Gamma_{\mathrm{I}}} \psi_{h} \vec{u}_{m} \cdot \vec{\nu} \mathrm{d} \Gamma-\int_{\Gamma_{\mathrm{I}}} \psi_{h} \vec{u}_{m h} \cdot \vec{\nu} \mathrm{d} \Gamma\right|}{\left\|\psi_{h}\right\|_{\mathrm{F}}}\right] .
$$

Using Lemma 3.1 and classical approximation results we get

$$
\inf _{\psi_{h} \in \mathrm{V}_{h}^{\mathrm{F}}}\left\|\varphi^{m}-\psi_{h}\right\|_{\mathrm{F}} \leq C h^{r}\left\|\varphi^{m}\right\|_{1+r, \Omega_{\mathrm{F}}} \leq C h^{r}\left\|\vec{u}_{m}\right\|_{1, \Omega_{\mathrm{S}}} .
$$

On the other hand,

$$
\begin{aligned}
\sup _{\psi_{h} \in \mathrm{V}_{h}^{\mathrm{F}}} \frac{\left|\int_{\Gamma_{\mathrm{I}}} \psi_{h} \vec{u}_{m} \cdot \vec{\nu} \mathrm{d} \Gamma-\int_{\Gamma_{\mathrm{I}}} \psi_{h} \vec{u}_{m h} \cdot \vec{\nu} \mathrm{d} \Gamma\right|}{\left\|\psi_{h}\right\|_{\mathrm{F}}} & \leq \sup _{\psi_{h} \in \mathrm{V}_{h}^{\mathrm{F}}} \frac{\left\|\psi_{h}\right\|_{0, \Gamma_{\mathrm{I}}}\left\|\vec{u}_{m} \cdot \vec{\nu}-\vec{u}_{m h} \cdot \vec{\nu}\right\|_{0, \Gamma_{\mathrm{I}}}}{\left\|\psi_{h}\right\|_{\mathrm{F}}} \\
& \leq C\left\|\vec{u}_{m}-\vec{u}_{m h}\right\|_{\mathrm{S}} \leq C h^{s}\left\|\vec{u}_{m}\right\|_{1+s, \Omega_{\mathrm{S}}} .
\end{aligned}
$$

and (ii) follows since $t=\min \{r, s\}$.

We define the finite dimensional space

$$
\mathbf{V}_{h}^{N, 2}=\left\langle\left\{\left(\varphi_{i h}, \overrightarrow{0}\right)\right\}_{i=1}^{N_{\mathrm{F}}} \cup\left\{\left(\varphi_{h}^{m}, \vec{u}_{m h}\right)\right\}_{m=1}^{N_{\mathrm{S}}}\right\rangle .
$$




\section{Modal Synthesis}

Taking into account the finite dimensional spaces $\mathbf{V}_{h}^{N, 1}$ and $\mathbf{V}_{h}^{N, 2}$, as defined in the previous sections, we can introduce the approximate coupled problem by modal synthesis.

$\mathbf{V P}_{h}^{N}$ : Find $\lambda_{h}^{N} \in \mathbb{R}$ and $(0, \overrightarrow{0}) \neq\left(\varphi_{h}, \vec{u}_{h}\right) \in \mathbf{V}_{h}^{N, 2}$, such that

$$
a\left(\left(\varphi_{h}, \vec{u}_{h}\right),\left(\psi_{h}, \vec{v}_{h}\right)\right)=\lambda_{h}^{N} b\left(\left(\varphi_{h}, \vec{u}_{h}\right),\left(\psi_{h}, \vec{v}_{h}\right)\right) \quad \forall\left(\psi_{h}, \vec{v}_{h}\right) \in \mathbf{V}_{h}^{N, 1}
$$

In the remaining of this section we deduce the matrix formulation of this spectral coupled problem (see [11] for more details).

Since $\left(\varphi_{h}, \vec{u}_{h}\right)$ belongs to $\mathbf{V}_{h}^{N, 2}$, we have

$$
\left(\varphi_{h}, \vec{u}_{h}\right)=\sum_{i=1}^{N_{\mathrm{F}}} \alpha_{i h}\left(\varphi_{i h}, \overrightarrow{0}\right)+\sum_{m=1}^{N_{\mathrm{S}}} \beta_{m h}\left(\varphi_{h}^{m}, \vec{u}_{m h}\right)=\left(\sum_{i=1}^{N_{\mathrm{F}}} \alpha_{i h} \varphi_{i h}+\sum_{m=1}^{N_{\mathrm{S}}} \beta_{m h} \varphi_{h}^{m}, \sum_{m=1}^{N_{\mathrm{S}}} \beta_{m h} \vec{u}_{m h}\right)
$$

for some real coefficients $\alpha_{i h}$ and $\beta_{m h}$ which are the unknowns of our problem. If we introduce this decomposition in $\mathbf{V P} \mathbf{P}_{h}^{N}$ and develop the bilinear forms therein, we obtain

$$
\begin{aligned}
& \sum_{i=1}^{N_{\mathrm{F}}} \alpha_{i h} \int_{\Omega_{\mathrm{F}}} \rho_{\mathrm{F}} \vec{\nabla} \varphi_{i h} \cdot \vec{\nabla} \psi_{h} \mathrm{~d} x+\sum_{m=1}^{N_{\mathrm{S}}} \beta_{m h} \int_{\Omega_{\mathrm{F}}} \rho_{\mathrm{F}} \vec{\nabla} \varphi_{h}^{m} \cdot \vec{\nabla} \psi_{h} \mathrm{~d} x+\sum_{m=1}^{N_{\mathrm{S}}} \beta_{m h} \int_{\Omega_{\mathrm{S}}} \sigma\left(\vec{u}_{m h}\right): \epsilon\left(\vec{v}_{h}\right) \mathrm{d} x \\
& -\sum_{m=1}^{N_{\mathrm{S}}} \beta_{m h} \int_{\Gamma_{\mathrm{I}}} \rho_{\mathrm{F}} \psi_{h} \vec{u}_{m h} \cdot \vec{\nu} \mathrm{d} \Gamma+\sum_{m=1}^{N_{\mathrm{S}}} \beta_{m h} \frac{\rho_{\mathrm{F}} c^{2}}{\left|\Omega_{\mathrm{F}}\right|} \int_{\Gamma_{\mathrm{I}}} \vec{u}_{m h} \cdot \vec{\nu} \mathrm{d} \Gamma \int_{\Gamma_{\mathrm{I}}} \vec{v}_{h} \cdot \vec{\nu} \mathrm{d} \Gamma \\
& =\lambda_{h}^{N}\left[\sum_{i=1}^{N_{\mathrm{F}}} \alpha_{i h} \int_{\Omega_{\mathrm{F}}} \frac{\rho_{\mathrm{F}}}{c^{2}} \varphi_{i h} \psi_{h} \mathrm{~d} x+\sum_{m=1}^{N_{\mathrm{S}}} \beta_{m h} \int_{\Omega_{\mathrm{F}}} \frac{\rho_{\mathrm{F}}}{c^{2}} \varphi_{h}^{m} \psi_{h} \mathrm{~d} x+\sum_{m=1}^{N_{\mathrm{S}}} \beta_{m h} \int_{\Omega_{\mathrm{S}}} \rho_{\mathrm{S}} \vec{u}_{m h} \cdot \vec{v}_{h} \mathrm{~d} x\right. \\
& \left.\quad+\sum_{i=1}^{N_{\mathrm{F}}} \alpha_{i h} \int_{\Gamma_{\mathrm{I}}} \rho_{\mathrm{F}} \varphi_{i h} \vec{v}_{h} \cdot \vec{\nu} \mathrm{d} \Gamma+\sum_{m=1}^{N_{\mathrm{S}}} \beta_{m h} \int_{\Gamma_{\mathrm{I}}} \rho_{\mathrm{F}} \varphi_{h}^{m} \vec{v}_{h} \cdot \vec{\nu} \mathrm{d} \Gamma\right] \quad \forall\left(\psi_{h}, \vec{v}_{h}\right) \in \mathbf{V}_{h}^{N, 1} .
\end{aligned}
$$

Now we take $\left(\psi_{h}, \vec{v}_{h}\right)=\left(\varphi_{j h}, \overrightarrow{0}\right), 1 \leq j \leq N_{\mathrm{F}}$, as test function. Taking into account (3.2), (3.3) and the definitions of $\varphi_{j h}$ and $\varphi_{h}^{m}$, we obtain

$$
\alpha_{j h} \mu_{j h}^{\mathrm{F}}=\lambda_{h}^{N}\left(\alpha_{j h} \frac{\mu_{j h}^{\mathrm{F}}}{\lambda_{j h}^{\mathrm{F}}}+\sum_{m=1}^{N_{\mathrm{S}}} \beta_{m h} \frac{1}{\lambda_{j h}^{\mathrm{F}}} \int_{\Omega_{\mathrm{F}}} \rho_{\mathrm{F}} \vec{\nabla} \varphi_{h}^{m} \cdot \vec{\nabla} \varphi_{j h} \mathrm{~d} x\right)
$$

what implies

$$
\alpha_{j h} \lambda_{j h}^{\mathrm{F}} \mu_{j h}^{\mathrm{F}}=\lambda_{h}^{N}\left(\alpha_{j h} \mu_{j h}^{\mathrm{F}}+\sum_{m=1}^{N_{\mathrm{S}}} \beta_{m h} \int_{\Omega_{\mathrm{F}}} \rho_{\mathrm{F}} \vec{\nabla} \varphi_{h}^{m} \cdot \vec{\nabla} \varphi_{j h} \mathrm{~d} x\right)
$$


Analogously, if we take $(\psi, \vec{v})=\left(0, \vec{u}_{n h}\right), 1 \leq n \leq N_{\mathrm{S}}$, as test function, use the equalities (3.4) and (3.5) and the definition of $\varphi_{h}^{m}$, we get

$$
\begin{aligned}
\beta_{n h} \lambda_{n h}^{\mathrm{S}} \mu_{n h}^{\mathrm{S}}+\sum_{m=1}^{N_{\mathrm{S}}} \beta_{m h} & \frac{\rho_{\mathrm{F}} c^{2}}{\left|\Omega_{\mathrm{F}}\right|} \int_{\Gamma_{\mathrm{I}}} \vec{u}_{m h} \cdot \vec{\nu} \mathrm{d} \Gamma \int_{\Gamma_{\mathrm{I}}} \vec{u}_{n h} \cdot \vec{\nu} \mathrm{d} \Gamma \\
& =\lambda_{h}^{N}\left[\beta_{n h} \mu_{n h}^{\mathrm{S}}+\sum_{i=1}^{N_{\mathrm{F}}} \alpha_{i h} \int_{\Omega_{\mathrm{F}}} \rho_{\mathrm{F}} \vec{\nabla} \varphi_{i h} \cdot \vec{\nabla} \varphi_{h}^{n} \mathrm{~d} x+\sum_{m=1}^{N_{\mathrm{S}}} \beta_{m h} \int_{\Omega_{\mathrm{F}}} \rho_{\mathrm{F}} \vec{\nabla} \varphi_{h}^{m} \cdot \vec{\nabla} \varphi_{h}^{n} \mathrm{~d} x\right] .
\end{aligned}
$$

From (5.1) and (5.2) we obtain the following matrix formulation of problem $\mathbf{V P}_{h}^{N}$,

$$
\left(\begin{array}{cc}
K_{11} & 0 \\
0 & K_{22}
\end{array}\right)\left(\begin{array}{c}
\alpha \\
\beta
\end{array}\right)=\lambda_{h}^{N}\left(\begin{array}{ll}
M_{11} & M_{12} \\
M_{12}^{t} & M_{22}
\end{array}\right)\left(\begin{array}{c}
\alpha \\
\beta
\end{array}\right)
$$

where

- $\alpha=\left(\alpha_{1 h}, \ldots, \alpha_{N_{\mathrm{F}} h}\right)$ and $\beta=\left(\beta_{1 h}, \ldots, \beta_{N_{\mathrm{S}}}\right)$,

- $\left(K_{11}\right)_{i j}=\delta_{i j} \lambda_{i h}^{\mathrm{F}} \mu_{i h}^{\mathrm{F}}, \quad 1 \leq i, j \leq N_{\mathrm{F}}$,

- $\left(K_{22}\right)_{m n}=\delta_{m n} \lambda_{m h}^{\mathrm{S}} \mu_{m h}^{\mathrm{S}}+\frac{\rho_{\mathrm{F}} c^{2}}{\left|\Omega_{\mathrm{F}}\right|} \int_{\Gamma_{\mathrm{I}}} \vec{u}_{m h} \cdot \vec{\nu} \mathrm{d} \Gamma \int_{\Gamma_{\mathrm{I}}} \vec{u}_{n h} \cdot \vec{\nu} \mathrm{d} \Gamma, \quad 1 \leq m, n \leq N_{\mathrm{S}}$,

- $\left(M_{11}\right)_{i j}=\delta_{i j} \mu_{i h}^{\mathrm{F}}, \quad 1 \leq i, j \leq N_{\mathrm{F}}$

- $\left(M_{12}\right)_{i n}=\int_{\Omega_{\mathrm{F}}} \rho_{\mathrm{F}} \vec{\nabla} \varphi_{i h} \cdot \vec{\nabla} \varphi_{h}^{n} \mathrm{~d} x, \quad 1 \leq i \leq N_{\mathrm{F}}, \quad 1 \leq n \leq N_{\mathrm{S}}$,

- $\left(M_{22}\right)_{m n}=\delta_{m n} \mu_{m h}^{\mathrm{S}}+\int_{\Omega_{\mathrm{F}}} \rho_{\mathrm{F}} \vec{\nabla} \varphi_{h}^{m} \cdot \vec{\nabla} \varphi_{h}^{n} \mathrm{~d} x, \quad 1 \leq m, n \leq N_{\mathrm{S}}$

We notice that both block matrices in (5.3) are symmetric. Typically, $N_{\mathrm{S}}$ and $N_{\mathrm{F}}$ are small numbers in applications. Hence, this is a low dimension eigenproblem.

We prove in the following lemma that the matrix on the left-hand side is positive definite. Then, the numerical solution of (5.3) is very simple.

Lemma 5.1. The matrix

$$
\left(\begin{array}{cc}
K_{11} & 0 \\
0 & K_{22}
\end{array}\right)
$$

as defined in (5.3), is positive definite.

Proof. For any $\alpha \in \mathbb{R}^{N_{\mathrm{F}}}$ and $\beta \in \mathbb{R}^{N_{\mathrm{S}}},(\alpha, \beta) \neq(0,0)$,

$$
\begin{aligned}
(\alpha \beta)\left(\begin{array}{cc}
K_{11} & 0 \\
0 & K_{22}
\end{array}\right)\left(\begin{array}{l}
\alpha \\
\beta
\end{array}\right)=\sum_{i=1}^{N_{\mathrm{F}}} \alpha_{i h}^{2} \lambda_{i h}^{\mathrm{F}} \mu_{i h}^{\mathrm{F}}+\sum_{m=1}^{N_{\mathrm{S}}} \beta_{m h}^{2} \lambda_{m h}^{\mathrm{S}} \mu_{m h}^{\mathrm{S}} & \\
& +\sum_{m, n=1}^{N_{\mathrm{S}}} \frac{\rho_{\mathrm{F}} c^{2}}{\left|\Omega_{\mathrm{F}}\right|} \beta_{m h} \beta_{n h} \int_{\Gamma_{\mathrm{I}}} \vec{u}_{m h} \cdot \vec{\nu} \mathrm{d} \Gamma \int_{\Gamma_{\mathrm{I}}} \vec{u}_{n h} \cdot \vec{\nu} \mathrm{d} \Gamma .
\end{aligned}
$$

The sum of the first two terms is clearly strictly positive, whereas the third one satisfies

$$
\sum_{m, n=1}^{N_{\mathrm{S}}} \frac{\rho_{\mathrm{F}} c^{2}}{\left|\Omega_{\mathrm{F}}\right|} \beta_{m h} \beta_{n h} \int_{\Gamma_{\mathrm{I}}} \vec{u}_{m h} \cdot \vec{\nu} \mathrm{d} \Gamma \int_{\Gamma_{\mathrm{I}}} \vec{u}_{n h} \cdot \vec{\nu} \mathrm{d} \Gamma=\frac{\rho_{\mathrm{F}} c^{2}}{\left|\Omega_{\mathrm{F}}\right|}\left(\int_{\Gamma_{\mathrm{I}}} \sum_{m=1}^{N_{\mathrm{S}}} \beta_{m h} \vec{u}_{m h} \cdot \vec{\nu} \mathrm{d} \Gamma\right)^{2} \geq 0 .
$$




\section{Preliminary theoretical Results}

We will use the results in [13] to estimate the error arising from the approximation of problem VP by the finite dimensional problem $\mathbf{V P}_{h}^{N}$. We prove the hypotheses of Theorems $6.3,6.4,6.6$, and 6.7 in [13]. Then we apply the results from this reference to our problem in Theorems 6.9 and 6.10 below.

To simplify the proofs, we assume that the uncoupled continuous and discrete eigenmodes are now normalized in such a way that

$$
\begin{array}{r}
\int_{\Omega_{\mathrm{F}}} \frac{\rho_{\mathrm{F}}}{c^{2}} \varphi_{i}^{2} \mathrm{~d} x=1, \quad \int_{\Omega_{\mathrm{F}}} \frac{\rho_{\mathrm{F}}}{c^{2}} \varphi_{i h}^{2} \mathrm{~d} x=1, \\
\int_{\Omega_{\mathrm{S}}} \rho_{\mathrm{S}}\left|\vec{u}_{m}\right|^{2} \mathrm{~d} x=1, \quad \int_{\Omega_{\mathrm{S}}} \rho_{\mathrm{S}}\left|\vec{u}_{m h}\right|^{2} \mathrm{~d} x=1 .
\end{array}
$$

Firstly we prove that $\mathbf{V}_{h}^{N, 2}$ approximates correctly $\mathbf{V}$, when $N_{\mathrm{F}}, N_{\mathrm{S}} \rightarrow \infty$ and $h \rightarrow 0$.

Lemma 6.1. The linear combinations of the functions $\left\{\left(\varphi_{i}, \overrightarrow{0}\right)\right\}_{i \geq 1} \cup\left\{\left(\varphi^{m}, \vec{u}_{m}\right)\right\}_{m \geq 1}$ are dense in $\mathbf{V}$.

Proof. Let $(\varphi, \vec{u})$ be an arbitrary element of $\mathbf{V}$.

Since $\left\{\frac{1}{\sqrt{\lambda_{m}^{\mathrm{S}}}} \vec{u}_{m}\right\}_{m \geq 1}$ is a Hilbert basis of $\mathbf{H}_{\Gamma_{\mathrm{D}}}^{1}\left(\Omega_{\mathrm{S}}\right)$ with respect to the norm $\|\cdot\|_{\mathrm{S}}$, we have

$$
\vec{u}=\sum_{m=1}^{\infty} \frac{1}{\lambda_{m}^{\mathrm{S}}}\left(\int_{\Omega_{\mathrm{S}}} \sigma(\vec{u}): \epsilon\left(\vec{u}_{m}\right) \mathrm{d} x\right) \vec{u}_{m}=\sum_{m=1}^{\infty}\left(\int_{\Omega_{\mathrm{S}}} \rho_{\mathrm{S}} \vec{u} \cdot \vec{u}_{m} \mathrm{~d} x\right) \vec{u}_{m}, \quad \text { in } \mathbf{H}^{1}\left(\Omega_{\mathrm{S}}\right)
$$

We denote $\beta_{m}=\int_{\Omega_{\mathrm{S}}} \rho_{\mathrm{S}} \vec{u} \cdot \vec{u}_{m} \mathrm{~d} x$.

Let $\varphi^{\vec{u} \cdot \vec{\nu}}$ be the static lifting associated to $\vec{u} \cdot \vec{\nu}$, as defined in SL. From the linearity and continuity of the static lifting operator, $\varphi^{\vec{u} \cdot \vec{\nu}}=\sum_{m=1}^{\infty} \beta_{m} \varphi^{m}$ in $\mathrm{H}^{1}\left(\Omega_{\mathrm{F}}\right)$.

Let $\alpha_{i}$ be the Fourier coefficients of $\varphi-\varphi^{\vec{u} \cdot \vec{\nu}}$ in the Hilbert basis $\left\{\frac{1}{\sqrt{\lambda_{i}^{F}}} \varphi_{i}\right\}_{i \geq 1}$ :

$$
\alpha_{i}=\frac{1}{\lambda_{i}^{\mathrm{F}}} \int_{\Omega_{\mathrm{F}}} \rho_{\mathrm{F}} \vec{\nabla}\left(\varphi-\varphi^{\vec{u} \cdot \vec{\nu}}\right) \cdot \vec{\nabla} \varphi_{i} \mathrm{~d} x=\int_{\Omega_{\mathrm{F}}} \frac{\rho_{\mathrm{F}}}{c^{2}}\left(\varphi-\varphi^{\vec{u} \cdot \vec{\nu}}\right) \varphi_{i} \mathrm{~d} x
$$

Then we have

$$
(\varphi, \vec{u})=\left(\varphi-\varphi^{\vec{u} \cdot \vec{v}}, \overrightarrow{0}\right)+\left(\varphi^{\vec{u} \cdot \vec{v}}, \vec{u}\right)=\sum_{i=1}^{\infty} \alpha_{i}\left(\varphi_{i}, \overrightarrow{0}\right)+\sum_{m=1}^{\infty} \beta_{m}\left(\varphi^{m}, \vec{u}_{m}\right) \quad \text { in } \quad \mathbf{V}
$$

Lemma 6.2. For any $\left\{\alpha_{i}\right\}_{i \geq 1}$ and $\left\{\beta_{m}\right\}_{m \geq 1}$ sequences of real numbers, the numerical series $\sum_{i=1}^{\infty} \alpha_{i}^{2} \lambda_{i}^{\mathrm{F}}$ and $\sum_{m=1}^{\infty} \beta_{m}^{2} \lambda_{m}^{\mathrm{S}}$ converge if and only if $\sum_{i=1}^{\infty} \alpha_{i}\left(\varphi_{i}, \overrightarrow{0}\right)$ and $\sum_{m=1}^{\infty} \beta_{m}\left(\varphi^{m}, \vec{u}_{m}\right)$ converge in $\mathbf{V}$.

Furthermore, there exist two strictly positive constants $C_{1}$ and $C_{2}$, such that

$$
C_{1}\left[\sum_{i=1}^{\infty} \alpha_{i}^{2} \lambda_{i}^{\mathrm{F}}+\sum_{m=1}^{\infty} \beta_{m}^{2} \lambda_{m}^{\mathrm{S}}\right] \leq\left\|\sum_{i=1}^{\infty} \alpha_{i}\left(\varphi_{i}, \overrightarrow{0}\right)+\sum_{m=1}^{\infty} \beta_{m}\left(\varphi^{m}, \vec{u}_{m}\right)\right\|_{\mathrm{V}}^{2} \leq C_{2}\left[\sum_{i=1}^{\infty} \alpha_{i}^{2} \lambda_{i}^{\mathrm{F}}+\sum_{m=1}^{\infty} \beta_{m}^{2} \lambda_{m}^{\mathrm{S}}\right]
$$


Proof. For any $\psi \in \stackrel{\mathrm{H}}{1}^{1}\left(\Omega_{\mathrm{F}}\right)$ and $\vec{v} \in \mathbf{H}_{\Gamma_{\mathrm{D}}}^{1}\left(\Omega_{\mathrm{S}}\right)$ we have

$$
\|\psi\|_{\mathrm{F}}^{2} \leq 2\left\|\psi+\varphi^{\vec{v} \cdot \vec{v}}\right\|_{\mathrm{F}}^{2}+2\left\|\varphi^{\vec{v} \cdot \vec{v}}\right\|_{\mathrm{F}}^{2} \leq 2\left\|\psi+\varphi^{\vec{v} \cdot \vec{\nu}}\right\|_{\mathrm{F}}^{2}+C\|\vec{v}\|_{\mathrm{S}}^{2}
$$

Then

$$
\|\psi\|_{\mathrm{F}}^{2}+\|\vec{v}\|_{\mathrm{S}}^{2} \leq \tilde{C}\left(\left\|\psi+\varphi^{\vec{v} \cdot \vec{v}}\right\|_{\mathrm{F}}^{2}+\|\vec{v}\|_{\mathrm{S}}^{2}\right)=\tilde{C}\left\|\left(\psi+\varphi^{\vec{v} \cdot \vec{v}}, \vec{v}\right)\right\|_{\mathrm{V}}^{2} .
$$

On the other hand, we have

$$
\left\|\left(\psi+\varphi^{\vec{v} \cdot \vec{\nu}}, \vec{v}\right)\right\|_{\mathrm{V}}^{2}=\left\|(\psi, \overrightarrow{0})+\left(\varphi^{\vec{v} \cdot \vec{\nu}}, \vec{v}\right)\right\|_{\mathrm{V}}^{2} \leq 2\|(\psi, \overrightarrow{0})\|_{\mathrm{V}}^{2}+2\left\|\left(\varphi^{\vec{v} \cdot \vec{\nu}}, \vec{v}\right)\right\|_{\mathrm{V}}^{2} \leq \tilde{\tilde{C}}\left(\|\psi\|_{\mathrm{F}}^{2}+\|\vec{v}\|_{\mathrm{S}}^{2}\right)
$$

From the definition of $\|\cdot\|_{\mathrm{F}}$ and $\|\cdot\|_{\mathrm{S}}$ and the normalization of the uncoupled eigenfunctions $\varphi_{i}$ and $\vec{u}_{m}$, $\left\|\varphi_{i}\right\|_{\mathrm{F}}=\sqrt{\lambda_{i}^{\mathrm{F}}}$ and $\left\|\vec{u}_{m}\right\|_{\mathrm{S}}=\sqrt{\lambda_{m}^{\mathrm{S}}}$. Then the lemma follows by taking $C_{1}=\frac{1}{\tilde{C}}, C_{2}=\tilde{\tilde{C}}, \psi=\sum_{i=1}^{I} \alpha_{i} \varphi_{i}$, $\vec{v}=\sum_{m=1}^{M} \beta_{m} \vec{u}_{m}$, and then letting $I, M \rightarrow \infty$.

As a direct consequence of Lemmas 3.2, 4.1, 6.1, and 6.2 we have the following:

Theorem 6.3. Let $(\varphi, \vec{u}) \in \mathbf{V}$, then

$$
\inf _{\left(\varphi_{h}, \vec{u}_{h}\right) \in \mathbf{V}_{h}^{N, 2}}\left\|(\varphi, \vec{u})-\left(\varphi_{h}, \vec{u}_{h}\right)\right\|_{\mathrm{V}} \rightarrow 0
$$

as $N_{\mathrm{F}}, N_{\mathrm{S}} \rightarrow \infty$ and $h \rightarrow 0$.

In the following theorem we prove that the bilinear form $a$ satisfies two inf-sup conditions.

Theorem 6.4. We have

$$
\begin{array}{r}
\inf _{\{(\varphi, \vec{u}) \in \mathrm{V}:\|(\varphi, \vec{u})\| \mathrm{V}=1\}} \sup _{\{(\psi, \vec{v}) \in \mathrm{V}:\|(\psi, \vec{v})\| \mathrm{V}=1\}}|a((\varphi, \vec{u}),(\psi, \vec{v}))|=\alpha>0, \\
\sup _{\{(\varphi, \vec{u}) \in \mathrm{V}:(\varphi, \vec{u}) \neq 0\}}|a((\varphi, \vec{u}),(\psi, \vec{v}))|>0 \quad \forall(\psi, \vec{v}) \in \mathbf{V},(\psi, \vec{v}) \neq(0, \overrightarrow{0}) .
\end{array}
$$

Proof. Let $(\varphi, \vec{u}) \in \mathbf{V}$. Let $\tilde{\varphi}$ be the only solution in $\stackrel{\circ}{H}^{1}\left(\Omega_{\mathrm{F}}\right)$ of the variational problem

$$
\int_{\Omega_{\mathrm{F}}} \vec{\nabla} \tilde{\varphi} \cdot \vec{\nabla} \psi \mathrm{d} x=\int_{\Omega_{\mathrm{F}}} \vec{\nabla} \varphi \cdot \vec{\nabla} \psi \mathrm{d} x-\int_{\Gamma_{\mathrm{I}}} \psi \vec{u} \cdot \vec{\nu} \mathrm{d} \Gamma \quad \forall \psi \in \stackrel{\circ}{\mathrm{H}}^{1}\left(\Omega_{\mathrm{F}}\right) .
$$

Then

$$
\int_{\Omega_{\mathrm{F}}} \vec{\nabla} \varphi \cdot \vec{\nabla} \psi \mathrm{d} x=\int_{\Omega_{\mathrm{F}}} \vec{\nabla} \tilde{\varphi} \cdot \vec{\nabla} \psi \mathrm{d} x+\int_{\Gamma_{\mathrm{I}}} \psi \vec{u} \cdot \vec{\nu} \mathrm{d} \Gamma \quad \forall \psi \in \stackrel{\circ}{\mathrm{H}}^{1}\left(\Omega_{\mathrm{F}}\right),
$$

and hence there exists a constant $C>0$ such that

$$
\|(\varphi, \vec{u})\|_{\mathrm{V}} \leq C\|(\tilde{\varphi}, \vec{u})\|_{\mathrm{V}} .
$$

Thus we have

$$
\begin{aligned}
\left|a\left(\frac{(\varphi, \vec{u})}{\|(\varphi, \vec{u})\|_{\mathrm{V}}}, \frac{(\tilde{\varphi}, \vec{u})}{\|(\tilde{\varphi}, \vec{u})\|_{\mathrm{V}}}\right)\right|= & \frac{1}{\|(\tilde{\varphi}, \vec{u})\|_{\mathrm{V}}\|(\varphi, \vec{u})\|_{\mathrm{V}}}\left(\int_{\Omega_{\mathrm{F}}} \rho_{\mathrm{F}}|\vec{\nabla} \tilde{\varphi}|^{2} \mathrm{~d} x+\int_{\Omega_{\mathrm{S}}} \sigma(\vec{u}): \epsilon(\vec{u}) \mathrm{d} x\right. \\
& \left.+\frac{\rho_{\mathrm{F}} c^{2}}{\left|\Omega_{\mathrm{F}}\right|}\left(\int_{\Gamma_{\mathrm{I}}} \vec{u} \cdot \vec{\nu} \mathrm{d} \Gamma\right)^{2}\right) \geq \frac{1}{\|(\varphi, \vec{u})\|_{\mathrm{V}}}\|(\tilde{\varphi}, \vec{u})\|_{\mathrm{V}} \geq \frac{1}{C}>0,
\end{aligned}
$$


what proves (6.1). Now, for any pair $(\tilde{\varphi}, \vec{u}) \in \mathbf{V}$, we consider $(\varphi, \vec{u}) \in \mathbf{V}$ with $\varphi$ the solution of $(6.3)$. Then

$$
a((\varphi, \vec{u}),(\tilde{\varphi}, \vec{u}))=\int_{\Omega_{\mathrm{F}}} \rho_{\mathrm{F}}|\vec{\nabla} \tilde{\varphi}|^{2} \mathrm{~d} x+\int_{\Omega_{\mathrm{S}}} \sigma(\vec{u}): \epsilon(\vec{u}) \mathrm{d} x+\frac{\rho_{\mathrm{F}} c^{2}}{\left|\Omega_{\mathrm{F}}\right|}\left(\int_{\Gamma_{\mathrm{I}}} \vec{u} \cdot \vec{\nu} \mathrm{d} \Gamma\right)^{2}>0,
$$

what proves (6.2).

Now we must prove two similar uniform inf-sup conditions on the discrete spaces. First, we show that $\mathbf{V}_{h}^{N, 2}$ and $\mathbf{V}_{h}^{N, 1}$ have the same dimension and then it is enough to prove one inf-sup discrete condition.

Lemma 6.5. $\operatorname{dim} \mathbf{V}_{h}^{N, 2}=\operatorname{dim} \mathbf{V}_{h}^{N, 1}=N_{\mathrm{S}}+N_{\mathrm{F}}$.

Proof. Clearly, $\operatorname{dim} \mathbf{V}_{h}^{N, 1}=N_{\mathrm{S}}+N_{\mathrm{F}}$. We will show that $\left\{\left(\varphi_{i h}, \overrightarrow{0}\right)\right\}_{i=1}^{N_{\mathrm{F}}} \cup\left\{\left(\varphi_{h}^{m}, \vec{u}_{m h}\right)\right\}_{m=1}^{N_{\mathrm{S}}}$ is an algebraic basis of $\mathbf{V}_{h}^{N, 2}$, what ends the proof.

Let us assume that

$$
(0, \overrightarrow{0})=\sum_{i=1}^{N_{\mathrm{F}}} \alpha_{i h}\left(\varphi_{i h}, \overrightarrow{0}\right)+\sum_{m=1}^{N_{\mathrm{S}}} \beta_{m h}\left(\varphi_{h}^{m}, \vec{u}_{m h}\right)=\left(\sum_{i=1}^{N_{\mathrm{F}}} \alpha_{i h} \varphi_{i h}+\sum_{m=1}^{N_{\mathrm{S}}} \beta_{m h} \varphi_{h}^{m}, \sum_{m=1}^{N_{\mathrm{S}}} \beta_{m h} \vec{u}_{m h}\right) .
$$

Then

$$
\overrightarrow{0}=\sum_{m=1}^{N_{\mathrm{S}}} \beta_{m h} \vec{u}_{m h} \Longrightarrow \beta_{m h}=0,1 \leq m \leq N_{\mathrm{S}}
$$

because the functions $\vec{u}_{m h}$ are linearly independent. Then (6.4) reduces to

$$
\sum_{i=1}^{N_{\mathrm{F}}} \alpha_{i h} \varphi_{i h}=0
$$

what implies $\alpha_{i h}=0,1 \leq i \leq N_{\mathrm{F}}$.

Theorem 6.6. The bilinear form a satisfies

$$
\inf _{\substack{\left(\varphi_{h}, \vec{u}_{h}\right) \in \mathbf{V}_{h}^{N, 2} \\\left\|\left(\varphi_{h}, \vec{u}_{h}\right)\right\|_{\mathrm{V}}=1}} \sup _{\substack{\left(\psi_{h}, \vec{v}_{h}\right) \in \mathbf{V}_{h}^{N, 1} \\\left\|\left(\psi_{h}, \vec{v}_{h}\right)\right\|_{\mathrm{V}}=1}}\left|a\left(\left(\varphi_{h}, \vec{u}_{h}\right),\left(\psi_{h}, \vec{v}_{h}\right)\right)\right| \geq \beta>0 .
$$

Proof. Firstly, proceeding as in Lemma 6.2, we prove that there exist two positive constants $C_{3}$ and $C_{4}$ such that

$$
\begin{aligned}
C_{3}\left(\sum_{i=1}^{N_{\mathrm{F}}} \alpha_{i h}^{2} \lambda_{i h}^{\mathrm{F}}+\sum_{m=1}^{N_{\mathrm{S}}} \beta_{m h}^{2} \lambda_{m h}^{\mathrm{S}}\right) & \leq\left\|\sum_{i=1}^{N_{\mathrm{F}}} \alpha_{i h}\left(\varphi_{i h}, \overrightarrow{0}\right)+\sum_{m=1}^{N_{\mathrm{S}}} \beta_{m h}\left(\varphi_{h}^{m}, \vec{u}_{m h}\right)\right\|_{\mathrm{V}}^{2} \\
& \leq C_{4}\left(\sum_{i=1}^{N_{\mathrm{F}}} \alpha_{i h}^{2} \lambda_{i h}^{\mathrm{F}}+\sum_{m=1}^{N_{\mathrm{S}}} \beta_{m h}^{2} \lambda_{m h}^{\mathrm{S}}\right) .
\end{aligned}
$$

Let $\left(\varphi_{h}, \vec{u}_{h}\right) \in \mathbf{V}_{h}^{N, 2}$ and $\alpha_{i h}, \beta_{m h}$ such that

$$
\left(\varphi_{h}, \vec{u}_{h}\right)=\sum_{i=1}^{N_{\mathrm{F}}} \alpha_{i h}\left(\varphi_{i h}, \overrightarrow{0}\right)+\sum_{m=1}^{N_{\mathrm{S}}} \beta_{m h}\left(\varphi_{h}^{m}, \vec{u}_{m h}\right)
$$


We define

$$
\left(\tilde{\varphi}_{h}, \vec{u}_{h}\right)=\sum_{i=1}^{N_{\mathrm{F}}} \alpha_{i h}\left(\varphi_{i h}, \overrightarrow{0}\right)+\sum_{m=1}^{N_{\mathrm{S}}} \beta_{m h}\left(0, \vec{u}_{m h}\right) \in \mathbf{V}_{h}^{N, 1}
$$

Then

$$
\begin{aligned}
a\left(\left(\varphi_{h}, \vec{u}_{h}\right),\left(\tilde{\varphi}_{h}, \vec{u}_{h}\right)\right)= & \sum_{i=1}^{N_{\mathrm{F}}} \alpha_{i h}^{2} \int_{\Omega_{\mathrm{F}}} \rho_{\mathrm{F}}\left|\vec{\nabla} \varphi_{i h}\right|^{2} \mathrm{~d} x+\sum_{m=1}^{N_{\mathrm{S}}} \beta_{m h}^{2} \int_{\Omega_{\mathrm{S}}} \sigma\left(\vec{u}_{m h}\right): \epsilon\left(\vec{u}_{m h}\right) \mathrm{d} x \\
& +\sum_{m=1}^{N_{\mathrm{S}}} \sum_{n=1}^{N_{\mathrm{S}}} \beta_{m h} \beta_{n h} \frac{\rho_{\mathrm{F}} c^{2}}{\left|\Omega_{\mathrm{F}}\right|} \int_{\Gamma_{\mathrm{I}}} \vec{u}_{m h} \cdot \vec{\nu} \mathrm{d} \Gamma \int_{\Gamma_{\mathrm{I}}} \vec{u}_{n h} \cdot \vec{\nu} \mathrm{d} \Gamma .
\end{aligned}
$$

Since

$$
\sum_{m=1}^{N_{\mathrm{S}}} \sum_{n=1}^{N_{\mathrm{S}}} \beta_{m h} \beta_{n h} \frac{\rho_{\mathrm{F}} c^{2}}{\left|\Omega_{\mathrm{F}}\right|} \int_{\Gamma_{\mathrm{I}}} \vec{u}_{m h} \cdot \vec{\nu} \mathrm{d} \Gamma \int_{\Gamma_{\mathrm{I}}} \vec{u}_{n h} \cdot \vec{\nu} \mathrm{d} \Gamma=\frac{\rho_{\mathrm{F}} c^{2}}{\left|\Omega_{\mathrm{F}}\right|}\left(\int_{\Gamma_{\mathrm{I}}} \vec{u}_{h} \cdot \vec{\nu} \mathrm{d} \Gamma\right)^{2} \geq 0,
$$

then, from (6.5), (6.6) and the definitions of $\varphi_{i h}$ and $\vec{u}_{m h}$, we have

$$
a\left(\left(\varphi_{h}, \vec{u}_{h}\right),\left(\tilde{\varphi}_{h}, \vec{v}_{h}\right)\right) \geq \sum_{i=1}^{N_{\mathrm{F}}} \alpha_{i h}^{2} \lambda_{i h}^{\mathrm{F}}+\sum_{m=1}^{N_{\mathrm{S}}} \beta_{m h}^{2} \lambda_{m h}^{\mathrm{S}} \geq \frac{1}{C_{4}}\left\|\left(\varphi_{h}, \vec{u}_{h}\right)\right\|_{\mathrm{V}}^{2} .
$$

We define the following operator associated with problem VP:

$$
T: \underset{(f, \vec{g})}{\mathbf{V}} \rightarrow \mathbf{V}(\varphi, \vec{u}): a((\varphi, \vec{u}),(\psi, \vec{v}))=b((f, \vec{g}),(\psi, \vec{v})) \quad \forall(\psi, \vec{v}) \in \mathbf{V} .
$$

Because of Theorem 6.4, $T$ is well defined and bounded (see, for instance, [1]). To prove the remaining hypotheses of [13] we will show that $T$ is a regularizing operator and then compact.

Theorem 6.7. Let $r>\frac{1}{2}$ and $s>0$ be the constants defined in Theorem 2.1. Then, for any $(f, \vec{g}) \in \mathbf{V}$, $T(f, \vec{g})=(\varphi, \vec{u}) \in \mathrm{H}^{1+r}\left(\Omega_{\mathrm{F}}\right) \times \mathbf{H}^{1+s}\left(\Omega_{\mathrm{S}}\right)$. Moreover,

$$
\|\varphi\|_{1+r, \Omega_{\mathrm{F}}}+\|\vec{u}\|_{1+s, \Omega_{\mathrm{S}}} \leq C\|(f, \vec{g})\|_{\mathrm{V}} .
$$

Proof. It follows by proceeding as in Lemma 6.2 of [2].

Then all the hypotheses of [13] are fulfilled and thus we can use the theory therein.

Let $\lambda$ be an eigenvalue of $\mathbf{V P}$ with multiplicity $k$. It is proved in [13] that there exist $k$ eigenvalues $\lambda_{h, 1}^{N}(\lambda), \ldots$, $\lambda_{h, k}^{N}(\lambda)$ of $\mathbf{V P}_{h}^{N}$, repeated according to their algebraic multiplicities, converging to $\lambda$ when $N_{\mathrm{F}}, N_{\mathrm{S}} \rightarrow \infty$ and $h \rightarrow 0$. Let $\mathbf{M}(\lambda)$ be the set of the eigenfunctions of $\mathbf{V P},(\varphi, \vec{u})$, associated to $\lambda$ with $\|(\varphi, \vec{u})\|_{\mathrm{V}}=1$ and $\mathbf{M}_{h}^{N}(\lambda)$ the set of the eigenfunctions of $\mathbf{V P}_{h}^{N},\left(\varphi_{h}, \vec{u}_{h}\right)$, associated to the eigenvalues $\lambda_{h, j}^{N}(\lambda), j=1, \ldots, k$, with $\left\|\left(\varphi_{h}, \vec{u}_{h}\right)\right\|_{\mathrm{V}}=1$. Let $\overline{\mathbf{M}}(\lambda)$ and $\overline{\mathbf{M}}_{h}^{N}(\lambda)$ be the linear spans of $\mathbf{M}(\lambda)$ and $\mathbf{M}_{h}^{N}(\lambda)$, respectively. Notice that, in our case, these two sets consist only of (not generalized) eigenfunctions, as a consequence of Theorem 2.1 and of the symmetry of problem $\mathbf{V P}_{h}^{N}$.

To apply the theory in [13] we also need to consider the "adjoint" operator $T_{*}$ defined by

$$
a\left((\varphi, \vec{u}), T_{*}(f, \vec{g})\right)=b((\varphi, \vec{u}),(f, \vec{g})) \quad \forall(\varphi, \vec{u}) \in \mathbf{V} .
$$


This is also a regularizing operator as shown in the following:

Lemma 6.8. Let $r$ and $s$ be the constants defined in Theorem 2.1. Then, for any $(f, \vec{g}) \in \mathbf{V},(\psi, \vec{v})=T_{*}(f, \vec{g}) \in$ $\mathrm{H}^{1+r}\left(\Omega_{\mathrm{F}}\right) \times \mathbf{H}^{1+s}\left(\Omega_{\mathrm{S}}\right)$ and

$$
\|\psi\|_{1+r, \Omega_{\mathrm{F}}}+\|\vec{v}\|_{1+s, \Omega_{\mathrm{S}}} \leq C\|(f, \vec{g})\|_{\mathrm{V}}
$$

Proof. Let $(\psi, \vec{v})$ be an adjoint eigenpair associated to $\lambda$. It is easy to prove that $\left(\rho_{\mathrm{F}} c^{2} \psi, \vec{v}\right)$ is a solution of the elastoacoustic problem written in terms of the fluid pressure (see [11]). Then, we conclude the proof from Lemma 6.2 in [2].

Since the spectrum of $T$ is real, the eigenvalues of $T_{*}$ coincide with the eigenvalues of $T$ with the same ascents (see for instance [1]). Now we are able to apply the results in [13]. To do this let $\mathbf{M}^{*}(\lambda)$ be the set of the eigenfunctions of $T_{*},(\psi, \vec{v})$, associated to $\lambda$ with $\|(\psi, \vec{v})\|_{\mathrm{V}}=1$.

Let $\widehat{\delta}(\mathbf{E}, \mathbf{F})$ denote the gap between two subspaces $\mathbf{E}$ and $\mathbf{F}$ of $\mathbf{V}$ (i.e., the symmetric distance in $\|\cdot\|_{\mathrm{V}}$ between subspaces, see [1] for a precise definition). Finally, let

$$
\begin{gathered}
\epsilon_{h}^{N}=\epsilon_{h}^{N}(\lambda)=\sup _{(\varphi, \vec{u}) \in \mathbf{M}(\lambda)} \inf _{\left(\varphi_{h}, \vec{u}_{h}\right) \in \mathbf{V}_{h}^{N, 2}}\left\|(\varphi, \vec{u})-\left(\varphi_{h}, \vec{u}_{h}\right)\right\|_{\mathrm{V}}, \\
\epsilon_{h}^{* N}=\epsilon_{h}^{* N}(\lambda)=\sup _{(\psi, \vec{v}) \in \mathbf{M}^{*}(\lambda)} \inf _{\left(\psi_{h}, \vec{v}_{h}\right) \in \mathbf{V}_{h}^{N, 1}}\left\|(\psi, \vec{v})-\left(\psi_{h}, \vec{v}_{h}\right)\right\|_{\mathrm{V}} .
\end{gathered}
$$

According to [13, Theorem 3], the following result holds.

Theorem 6.9. There exists a constant $C$ such that

$$
\delta\left(\overline{\mathbf{M}}(\lambda), \overline{\mathbf{M}_{h}^{N}}(\lambda)\right) \leq C \epsilon_{h}^{N}
$$

Moreover, since the ascent of any eigenvalue of VP is one, by applying Theorem 2 of [13] we get:

Theorem 6.10. There exists a constant $C$ such that

$$
\left|\lambda-\lambda_{h, j}^{N}\right| \leq C \epsilon_{h}^{N} \epsilon_{h}^{* N} .
$$

In the next section we give estimates of $\epsilon_{h}^{N}$ and $\epsilon_{h}^{* N}$. These estimates prove that, for each pair $N=\left(N_{\mathrm{F}}, N_{\mathrm{S}}\right)$, there exists $h_{N}$ such that $\epsilon_{h_{N}}^{N}, \epsilon_{h_{N}}^{* N} \rightarrow 0$ as $N_{\mathrm{F}}, N_{\mathrm{S}} \rightarrow \infty$. Then, according to the previous theorems, we have convergence for eigenvalues and eigenfunctions.

\section{Spectral Convergence}

Let $\lambda$ be an eigenvalue of VP with (finite) multiplicity $k$. Our aim is to bound $\epsilon_{h}^{N}(\lambda)$. $\operatorname{Since} \mathbf{M}(\lambda) \operatorname{has}$ exactly $k$ elements, we just need to bound the distance of any of these elements to $\mathbf{V}_{h}^{N, 2}$.

Let $(\varphi, \vec{u})$ be an eigenfunction of the continuous problem VP associated to the eigenvalue $\lambda$, with $\|(\varphi, \vec{u})\|_{\mathrm{V}}=$ 1. According to Lemma 6.1, we can write

$$
(\varphi, \vec{u})=\sum_{i=1}^{\infty} \alpha_{i}\left(\varphi_{i}, \overrightarrow{0}\right)+\sum_{m=1}^{\infty} \beta_{m}\left(\varphi^{m}, \vec{u}_{m}\right),
$$

with $\alpha_{i}=\int_{\Omega_{\mathrm{F}}} \frac{\rho_{\mathrm{F}}}{c^{2}}\left(\varphi-\varphi^{\vec{u} \cdot \vec{\nu}}\right) \varphi_{i} \mathrm{~d} x$ and $\beta_{m}=\int_{\Omega_{\mathrm{S}}} \rho_{\mathrm{S}} \vec{u} \cdot \vec{u}_{m} \mathrm{~d} x$. Let $\left(\tilde{\varphi_{h}}, \tilde{\vec{u}}_{h}\right) \in \mathbf{V}_{h}^{N, 2}$ defined by

$$
\left(\tilde{\varphi_{h}}, \tilde{\vec{u}}_{h}\right)=\sum_{i=1}^{N_{\mathrm{F}}} \alpha_{i}\left(\varphi_{i h}, \overrightarrow{0}\right)+\sum_{m=1}^{N_{\mathrm{S}}} \beta_{m}\left(\varphi^{m}, \vec{u}_{m}\right) .
$$


Then we have

$$
\begin{aligned}
& \inf _{\left(\varphi_{h}, \vec{u}_{h}\right) \in \mathbf{V}_{h}^{N, 2}}\left\|(\varphi, \vec{u})-\left(\varphi_{h}, \vec{u}_{h}\right)\right\|_{\mathrm{V}} \leq\left\|(\varphi, \vec{u})-\left(\tilde{\varphi}_{h}, \tilde{\vec{u}}_{h}\right)\right\|_{\mathrm{V}} \\
& \leq\left\|\sum_{i=1}^{N_{\mathrm{F}}} \alpha_{i}\left(\varphi_{i}-\varphi_{i h}, \overrightarrow{0}\right)+\sum_{m=1}^{N_{\mathrm{S}}} \beta_{m}\left(\varphi^{m}-\varphi_{h}^{m}, \vec{u}_{m}-\vec{u}_{m h}\right)\right\|_{\mathrm{V}}+\left\|\sum_{i=N_{\mathrm{F}}+1}^{\infty} \alpha_{i}\left(\varphi_{i}, \overrightarrow{0}\right)+\sum_{m=N_{\mathrm{S}}+1}^{\infty} \beta_{m}\left(\varphi^{m}, \vec{u}_{m}\right)\right\|_{\mathrm{V}}
\end{aligned}
$$

Regarding the second term, from Lemma 6.2 we have

$$
\left\|\sum_{i=N_{\mathrm{F}}+1}^{\infty} \alpha_{i}\left(\varphi_{i}, \overrightarrow{0}\right)+\sum_{m=N_{\mathrm{S}}+1}^{\infty} \beta_{m}\left(\varphi^{m}, \vec{u}_{m}\right)\right\|_{\mathrm{V}} \leq\left(C_{2}\right)^{\frac{1}{2}}\left[\sum_{i=N_{\mathrm{F}}+1}^{\infty} \alpha_{i}^{2} \lambda_{i}^{\mathrm{F}}+\sum_{m=N_{\mathrm{S}}+1}^{\infty} \beta_{m}^{2} \lambda_{m}^{\mathrm{S}}\right]^{\frac{1}{2}}
$$

In the following two lemmas we study each of the series on the right-hand side of this inequality.

Lemma 7.1. There holds

$$
\sum_{i=N_{\mathrm{F}}+1}^{\infty} \alpha_{i}^{2} \lambda_{i}^{\mathrm{F}} \leq\left(\lambda_{N_{\mathrm{F}}+1}^{\mathrm{F}}\right)^{-1} \sum_{i=N_{\mathrm{F}}+1}^{\infty} \alpha_{i}^{2}\left(\lambda_{i}^{\mathrm{F}}\right)^{2}<\infty
$$

Proof. Because of the definition of VP and SL, $-\Delta \varphi,-\Delta \varphi^{\vec{u} \cdot \vec{\nu}} \in \mathrm{L}^{2}\left(\Omega_{\mathrm{F}}\right),\left.\frac{\partial}{\partial \nu} \varphi\right|_{\Gamma_{\mathrm{I}}}=\left.\frac{\partial}{\partial \nu} \varphi^{\vec{u} \cdot \vec{\nu}}\right|_{\Gamma_{\mathrm{I}}}=\left.\vec{u} \cdot \vec{\nu}\right|_{\Gamma_{\mathrm{I}}}$. Then, if we consider the inner product in $\mathrm{L}^{2}\left(\Omega_{\mathrm{F}}\right)$

$$
\left(\psi_{1}, \psi_{2}\right)_{\mathrm{F}}:=\int_{\Omega_{\mathrm{F}}} \frac{\rho_{\mathrm{F}}}{c^{2}} \psi_{1} \psi_{2} \mathrm{~d} x, \forall \psi_{1}, \psi_{2} \in \mathrm{L}^{2}\left(\Omega_{\mathrm{F}}\right),
$$

we obtain

$$
\left(-\Delta\left(\varphi-\varphi^{\vec{u} \cdot \vec{\nu}}\right), \varphi_{i}\right)_{\mathrm{F}}=\int_{\Omega_{\mathrm{F}}} \frac{\rho_{\mathrm{F}}}{c^{2}} \vec{\nabla}\left(\varphi-\varphi^{\vec{u} \cdot \vec{\nu}}\right) \cdot \vec{\nabla} \varphi_{i} \mathrm{~d} x=\frac{1}{c^{2}} \lambda_{i}^{\mathrm{F}} \int_{\Omega_{\mathrm{F}}} \frac{\rho_{\mathrm{F}}}{c^{2}}\left(\varphi-\varphi^{\vec{u} \cdot \vec{\nu}}\right) \varphi_{i} \mathrm{~d} x=\frac{1}{c^{2}} \alpha_{i} \lambda_{i}^{\mathrm{F}}
$$

where the first equality is obtained by integrating by parts, the second one using that $\varphi_{i}$ is an eigenfunction of $\mathbf{V P}^{F}$ with associated eigenvalue $\lambda_{i}^{\mathrm{F}}$, and the third one comes from the definition of $\alpha_{i}$.

Since $\left\{\varphi_{i}\right\}_{i \geq 1}$ is a Hilbert basis of $\mathrm{L}^{2}\left(\Omega_{\mathrm{F}}\right)$ endowed with the norm induced by $(\cdot, \cdot)_{F}$, then

$$
\sum_{i \geq 1} \alpha_{i}^{2}\left(\lambda_{i}^{\mathrm{F}}\right)^{2}=c^{4} \sum_{i \geq 1}\left(-\Delta\left(\varphi-\varphi^{\vec{u} \cdot \vec{\nu}}\right), \varphi_{i}\right)<\infty
$$

Finally, since we assume that the eigenvalues $\lambda_{i}^{\mathrm{F}}$ are numbered in increasing order, we get

$$
\sum_{i=N_{\mathrm{F}}+1}^{\infty} \alpha_{i}^{2} \lambda_{i}^{\mathrm{F}}=\sum_{i=N_{\mathrm{F}}+1}^{\infty}\left(\lambda_{i}^{\mathrm{F}}\right)^{-1} \alpha_{i}^{2}\left(\lambda_{i}^{\mathrm{F}}\right)^{2} \leq\left(\lambda_{N_{\mathrm{F}}+1}^{\mathrm{F}}\right)^{-1} \sum_{i=N_{\mathrm{F}}+1}^{\infty} \alpha_{i}^{2}\left(\lambda_{i}^{\mathrm{F}}\right)^{2}<\infty .
$$

To bound the second series in (7.2) there is a fundamental difference. As we have shown, $\alpha_{i}$ are the Fourier coefficients of $\varphi-\varphi^{\vec{u} \cdot \vec{\nu}}$. This function belongs to the domain of the Laplace operator with homogeneous Neumann boundary conditions, which is the operator diagonalized by the eigenfunctions $\left\{\varphi_{i}\right\}_{i>1}$. Instead, in the solid, $\beta_{m}$ are the Fourier coefficients of $\vec{u}$. Since, in general, $\sigma(\vec{u}) \cdot \vec{\nu} \neq 0$ on $\Gamma_{\mathrm{I}}, \vec{u}$ is not in the domain of the operator with eigenfunctions $\left\{\vec{u}_{m}\right\}_{m \geq 1}$. This leads to a lost of accuracy in the order of convergence. 
Lemma 7.2. Let $s$ be the regularity constant in Theorem 2.1. Then

$$
\sum_{m=N_{\mathrm{S}}+1}^{\infty} \beta_{m}^{2} \lambda_{m}^{\mathrm{S}} \leq\left(\lambda_{N_{\mathrm{S}}+1}^{\mathrm{S}}\right)^{-\hat{s}} \sum_{m=N_{\mathrm{S}}+1}^{\infty} \beta_{m}^{2}\left(\lambda_{m}^{\mathrm{S}}\right)^{1+\hat{s}}<\infty \quad \forall \hat{s}<\min \left\{\frac{1}{2}, s\right\} .
$$

Proof. Let

$$
\mathbf{H}_{\mathrm{B}}^{2}\left(\Omega_{\mathrm{S}}\right)=\left\{\vec{v} \in \mathbf{H}^{2}\left(\Omega_{\mathrm{S}}\right):\left.\vec{v}\right|_{\Gamma_{\mathrm{D}}}=\overrightarrow{0},\left.\sigma(\vec{v}) \cdot \vec{\nu}\right|_{\Gamma_{\mathrm{N}} \cup \Gamma_{\mathrm{I}}}=0\right\}
$$

In [20] (where the results in [10] are generalized to polygonal domains with mixed boundary conditions) it is proved that the boundary conditions involving derivatives are lost below $\mathrm{H}^{\frac{3}{2}}\left(\Omega_{\mathrm{S}}\right)$, namely,

$$
\left[\mathbf{H}_{\mathrm{B}}^{2}\left(\Omega_{\mathrm{S}}\right), \mathbf{L}^{2}\left(\Omega_{\mathrm{S}}\right)\right]_{\theta}=\left\{\vec{v} \in \mathbf{H}^{2(1-\theta)}\left(\Omega_{\mathrm{S}}\right):\left.\vec{v}\right|_{\Gamma_{\mathrm{D}}}=\overrightarrow{0}\right\}, \text { if } \theta>\frac{1}{4}
$$

We denote by $\mathbf{A}$ the linear elasticity operator with homogeneous Dirichlet boundary conditions on $\Gamma_{\mathrm{D}}$ and homogeneous Neumann boundary conditions on $\Gamma_{\mathrm{N}} \cup \Gamma_{\mathrm{I}}$. Let $\mathbf{D}(\mathbf{A})$ be its domain. It is clear that $\mathbf{H}_{\mathrm{B}}^{2}\left(\Omega_{\mathrm{S}}\right) \subset$ $\mathbf{D}(\mathbf{A})$, then $\left[\mathbf{H}_{\mathrm{B}}^{2}\left(\Omega_{\mathrm{S}}\right), \mathbf{L}^{2}\left(\Omega_{\mathrm{S}}\right)\right]_{\theta} \subset\left[\mathbf{D}(\mathbf{A}), \mathbf{L}^{2}\left(\Omega_{\mathrm{S}}\right)\right]_{\theta}, \forall \theta \in(0,1)$. The intermediate spaces between $\mathbf{D}(\mathbf{A})$ and $\mathbf{L}^{2}\left(\Omega_{\mathrm{S}}\right)$ can be characterized, according to [14], by

$$
\left[\mathbf{D}(\mathbf{A}), \mathbf{L}^{2}\left(\Omega_{\mathrm{S}}\right)\right]_{\theta}=\mathbf{D}\left(\mathbf{A}^{1-\theta}\right)=\left\{\vec{v} \in \mathbf{L}^{2}\left(\Omega_{\mathrm{S}}\right): \sum_{m=1}^{\infty}\left(\lambda_{m}^{\mathrm{S}}\right)^{2(1-\theta)}\left(\vec{v}, \vec{u}_{m}\right)_{S}^{2}<\infty\right\}, \theta \in(0,1),
$$

where $\left(\vec{v}, \vec{u}_{m}\right)_{S}=\int_{\Omega_{\mathrm{S}}} \rho_{\mathrm{S}} \vec{v} \cdot \vec{u}_{m} \mathrm{~d} x$.

We distinguish two possibilities:

- $s \geq \frac{1}{2}$. Then $\vec{u} \in \mathbf{H}^{\frac{3}{2}}\left(\Omega_{\mathrm{S}}\right)$. Since $\left.\vec{u}\right|_{\Gamma_{\mathrm{D}}}=\overrightarrow{0}$ but $\left.\sigma(\vec{u}) \cdot \vec{\nu}\right|_{\Gamma_{\mathrm{N}} \cup \Gamma_{\mathrm{I}}} \neq 0$, then

$$
\vec{u} \in\left[\mathbf{H}_{\mathrm{B}}^{2}\left(\Omega_{\mathrm{S}}\right), \mathbf{L}^{2}\left(\Omega_{\mathrm{S}}\right)\right]_{\frac{1}{4}+\epsilon} \Rightarrow \sum_{m=1}^{\infty}\left(\lambda_{m}^{\mathrm{S}}\right)^{\frac{3}{2}-2 \epsilon}\left(\int_{\Omega_{\mathrm{S}}} \rho_{\mathrm{S}} \vec{u} \cdot \vec{u}_{m} \mathrm{~d} x\right)^{2}<\infty, \epsilon>0 .
$$

- $s<\frac{1}{2}$. In this case $\vec{u} \in \mathbf{H}^{1+s}\left(\Omega_{\mathrm{S}}\right)=\mathbf{H}^{2\left(1-\frac{1-s}{2}\right)}\left(\Omega_{\mathrm{S}}\right)$. Then

$$
\vec{u} \in\left[\mathbf{H}_{\mathrm{B}}^{2}\left(\Omega_{\mathrm{S}}\right), \mathbf{L}^{2}\left(\Omega_{\mathrm{S}}\right)\right]_{\frac{1-s}{2}} \Rightarrow \sum_{m=1}^{\infty}\left(\lambda_{m}^{\mathrm{S}}\right)^{1+s}\left(\int_{\Omega_{\mathrm{S}}} \rho_{\mathrm{S}} \vec{u} \cdot \vec{u}_{m} \mathrm{~d} x\right)^{2}<\infty .
$$

Thus, in both cases, $\sum_{m=1}^{\infty} \beta_{m}^{2}\left(\lambda_{m}^{\mathrm{S}}\right)^{1+\hat{s}}<\infty$, with $\hat{s}$ as defined above. Then

$$
\sum_{m=N_{\mathrm{S}}+1}^{\infty} \beta_{m}^{2} \lambda_{m}^{\mathrm{S}} \leq\left(\lambda_{N_{\mathrm{S}}+1}^{\mathrm{S}}\right)^{-\hat{s}} \sum_{m=N_{\mathrm{S}}+1}^{\infty} \beta_{m}^{2}\left(\lambda_{m}^{\mathrm{S}}\right)^{1+\hat{s}}<\infty .
$$

In the next two lemmas we will bound the first term in (7.1).

Lemma 7.3. Let $r$ be the regularity constant in Theorem 2.1. Then

$$
\left\|\sum_{i=1}^{N_{\mathrm{F}}} \alpha_{i}\left(\varphi_{i}-\varphi_{i h}, \overrightarrow{0}\right)\right\|_{\mathrm{V}} \leq C h^{r} \sqrt{N_{\mathrm{F}}}
$$

where $C$ is a constant depending on $\lambda$ but independent of $N_{\mathrm{F}}$ and $h$. 
Proof. By applying Lemmas 3.1 and 3.2 and having into account the normalization of $\varphi_{i}$, we have

$$
\left\|\varphi_{i}-\varphi_{i h}\right\|_{\mathrm{F}} \leq C h^{r}\left\|\varphi_{i}\right\|_{1+r, \Omega_{\mathrm{F}}} \leq C h^{r} \lambda_{i}^{\mathrm{F}} .
$$

Then

$$
\left\|\sum_{i=1}^{N_{\mathrm{F}}} \alpha_{i}\left(\varphi_{i}-\varphi_{i h}, \overrightarrow{0}\right)\right\|_{\mathrm{V}} \leq \sum_{i=1}^{N_{\mathrm{F}}}\left|\alpha_{i}\right|\left\|\varphi_{i}-\varphi_{i h}\right\|_{\mathrm{F}} \leq C h^{r} \sum_{i=1}^{N_{\mathrm{F}}}\left|\alpha_{i}\right| \lambda_{i}^{\mathrm{F}} \leq C h^{r} \sqrt{N_{\mathrm{F}}}\left(\sum_{n=1}^{N_{\mathrm{F}}} \alpha_{i}^{2}\left(\lambda_{i}^{\mathrm{F}}\right)^{2}\right)^{\frac{1}{2}} .
$$

Now, by reasoning as in Lemma 7.1,

$$
\left(\sum_{n=1}^{N_{\mathrm{F}}} \alpha_{i}^{2}\left(\lambda_{i}^{\mathrm{F}}\right)^{2}\right)^{\frac{1}{2}} \leq\left(\sum_{n=1}^{\infty} \alpha_{i}^{2}\left(\lambda_{i}^{\mathrm{F}}\right)^{2}\right)^{\frac{1}{2}}=\left\|-\Delta\left(\varphi-\varphi^{\vec{u} \cdot \vec{\nu}}\right)\right\|_{0, \Omega_{\mathrm{F}}} .
$$

Since $-\Delta \varphi=\lambda \varphi$ and $\Delta \varphi^{\vec{u} \cdot \vec{\nu}}=\frac{1}{\Omega_{\mathrm{F}}} \int_{\Gamma_{\mathrm{I}}} \vec{u} \cdot \vec{\nu} \mathrm{d} \Gamma$ in $\Omega_{\mathrm{F}}$,

$$
\left\|-\Delta\left(\varphi-\varphi^{\vec{u} \cdot \vec{v}}\right)\right\|_{0, \Omega_{\mathrm{F}}} \leq C \lambda\|(\varphi, \vec{u})\|_{V} .
$$

We have $(\varphi, \vec{u}) \in \mathbf{M}(\lambda)$, what implies $\|(\varphi, \vec{u})\|_{V}=1$. Then, joining (7.3), (7.4), and (7.5), we conclude the proof.

Lemma 7.4. Let $s$ and $t$ be the constants defined in Lemma 4.1 and $\hat{s}$ as defined in Lemma 7.2. Then

$$
\left\|\sum_{m=1}^{N_{\mathrm{S}}} \beta_{m}\left(\varphi^{m}-\varphi_{h}^{m}, \vec{u}_{m}-\vec{u}_{m h}\right)\right\|_{\mathrm{V}} \leq C h^{t}\left(\lambda_{N_{\mathrm{S}}}^{\mathrm{S}}\right)^{\frac{1-\hat{s}}{2}} \sqrt{N_{\mathrm{S}}}
$$

Proof. We have

$$
\left\|\sum_{m=1}^{N_{\mathrm{S}}} \beta_{m}\left(\varphi^{m}-\varphi_{h}^{m}, \vec{u}_{m}-\vec{u}_{m h}\right)\right\|_{\mathrm{V}} \leq \sum_{m=1}^{N_{\mathrm{S}}}\left|\beta_{m}\right|\left\|\varphi^{m}-\varphi_{h}^{m}\right\|_{\mathrm{F}}+\sum_{m=1}^{N_{\mathrm{S}}}\left|\beta_{m}\right|\left\|\vec{u}_{m}-\vec{u}_{m h}\right\|_{\mathrm{S}} .
$$

Using Lemma 4.1 we have

$$
\sum_{m=1}^{N_{\mathrm{S}}}\left|\beta_{m}\right|\left\|\varphi^{m}-\varphi_{h}^{m}\right\|_{\mathrm{F}} \leq C h^{t} \sum_{m=1}^{N_{\mathrm{S}}}\left|\beta_{m}\right|\left\|\vec{u}_{m}\right\|_{1+s, \Omega_{\mathrm{S}}} \leq C h^{t} \sum_{m=1}^{N_{\mathrm{S}}}\left|\beta_{m}\right| \lambda_{m}^{\mathrm{S}} .
$$

Analogously, using Lemma 3.2, we obtain

$$
\sum_{m=1}^{N_{\mathrm{S}}}\left|\beta_{m}\right|\left\|\vec{u}_{m}-\vec{u}_{m h}\right\|_{\mathrm{S}} \leq C h^{s} \sum_{m=1}^{N_{\mathrm{S}}}\left|\beta_{m}\right| \lambda_{m}^{\mathrm{S}} \leq C h^{t} \sum_{m=1}^{N_{\mathrm{S}}}\left|\beta_{m}\right| \lambda_{m}^{\mathrm{S}}
$$

Thus the following inequality follows:

$$
\left\|\sum_{m=1}^{N_{\mathrm{S}}} \beta_{m}\left(\varphi^{m}-\varphi_{h}^{m}, \vec{u}_{m}-\vec{u}_{m h}\right)\right\|_{\mathrm{V}} \leq C h^{t} \sqrt{N_{\mathrm{S}}}\left(\sum_{m=1}^{N_{\mathrm{S}}} \beta_{m}^{2}\left(\lambda_{m}^{\mathrm{S}}\right)^{2}\right)^{\frac{1}{2}} \leq C h^{t} \sqrt{N_{\mathrm{S}}}\left(\lambda_{N_{\mathrm{S}}}^{\mathrm{S}}\right)^{\frac{1-\hat{s}}{2}}\left(\sum_{m=1}^{N_{\mathrm{S}}} \beta_{m}^{2}\left(\lambda_{m}^{\mathrm{S}}\right)^{1+\hat{s}}\right)^{\frac{1}{2}} .
$$


It is proved, in Lemma 7.2, that $\vec{u} \in\left[\mathbf{D}(\mathbf{A}), \mathbf{L}^{2}\left(\Omega_{\mathrm{S}}\right)\right]_{\frac{1-\hat{s}}{2}}$. Moreover, the norm of $\vec{u}$ in this space can be defined as $\left(\sum_{m=1}^{\infty} \beta_{m}^{2}\left(\lambda_{m}^{\mathrm{S}}\right)^{1+\hat{s}}\right)^{\frac{1}{2}}$. Then, since the inclusions

$$
\mathbf{H}^{1+\hat{s}}\left(\Omega_{\mathrm{S}}\right) \subset\left[\mathbf{H}_{B}^{2}\left(\Omega_{\mathrm{S}}\right), \mathbf{L}^{2}\left(\Omega_{\mathrm{S}}\right)\right]_{\frac{1-\hat{s}}{2}} \subset\left[\mathbf{D}(\mathbf{A}), \mathbf{L}^{2}\left(\Omega_{\mathrm{S}}\right)\right]_{\frac{1-\hat{s}}{2}}
$$

hold, and $\hat{s} \leq s$,

$$
\left(\sum_{m=1}^{\infty} \beta_{m}^{2}\left(\lambda_{m}^{\mathrm{S}}\right)^{1+\hat{s}}\right)^{\frac{1}{2}} \leq C\|\vec{u}\|_{1+\hat{s}, \Omega_{\mathrm{S}}} \leq C\|\vec{u}\|_{1+s, \Omega_{\mathrm{S}}} .
$$

Now, since $\vec{u}$ is an eigenfunction of VP for the eigenvalue $\lambda$,

$$
\|\vec{u}\|_{1+s, \Omega_{\mathrm{S}}} \leq C \lambda\|\vec{u}\|_{1, \Omega_{\mathrm{S}}} \leq C
$$

where $C$ is a constant depending on $\lambda$.

Joining (7.6), (7.7), and (7.8) we conclude the proof.

As a consequence of the previous lemmas we can bound $\epsilon_{h}^{N}$.

Theorem 7.5. For each pair $N=\left(N_{\mathrm{F}}, N_{\mathrm{S}}\right)$ there exists $h_{N}$ such that

$$
\lim _{N_{\mathrm{F}}, N_{\mathrm{S}} \rightarrow \infty} \epsilon_{h_{N}}^{N}=0 .
$$

Proof. We have an error estimate for $\epsilon_{h}^{N}$, consequence of inequality (7.1) and of the previous lemmas:

$$
\begin{aligned}
\epsilon_{h}^{N} \leq & C\left(\left(\lambda_{N_{\mathrm{F}}+1}^{\mathrm{F}}\right)^{-\frac{1}{2}}\left(\sum_{i=N_{\mathrm{F}}+1}^{\infty} \alpha_{i}^{2}\left(\lambda_{i}^{\mathrm{F}}\right)^{2}\right)^{\frac{1}{2}}+\left(\lambda_{N_{\mathrm{S}}+1}^{\mathrm{S}}\right)^{-\frac{s}{2}}\left(\sum_{m=N_{\mathrm{S}}+1}^{\infty} \beta_{m}^{2}\left(\lambda_{m}^{\mathrm{S}}\right)^{1+\hat{s}}\right)^{\frac{1}{2}}\right) \\
& +C h^{t}\left(\sqrt{N_{\mathrm{F}}}+\left(\lambda_{N_{\mathrm{S}}}^{\mathrm{S}}\right)^{\frac{1-\hat{s}}{2}} \sqrt{N_{\mathrm{S}}}\right) .
\end{aligned}
$$

The first term in the previous expression goes to zero when $N_{\mathrm{F}}, N_{\mathrm{S}} \rightarrow \infty$. Referring to the second term, given any pair $N=\left(N_{\mathrm{F}}, N_{\mathrm{S}}\right)$, we can choose $h_{N}>0$ such that

$$
\lim _{N_{\mathrm{F}}, N_{\mathrm{S}} \rightarrow \infty} h_{N}^{t}\left(\sqrt{N_{\mathrm{F}}}+\left(\lambda_{N_{\mathrm{S}}}^{\mathrm{S}}\right)^{\frac{1-3}{2}} \sqrt{N_{\mathrm{S}}}\right)=0
$$

In the next Theorem we bound $\epsilon_{h}^{* N}(\lambda)$. Let $(\psi, \vec{v})$ be an adjoint eigenpair associated to $\lambda$. We must bound the distance of $(\psi, \vec{v})$ to the finite dimensional space

$$
\mathbf{V}_{h}^{N, 1}=\left\langle\left\{\left(\varphi_{i h}, \overrightarrow{0}\right)\right\}_{i=1}^{N_{\mathrm{F}}} \cup\left\{\left(0, \vec{u}_{m h}\right)\right\}_{m=1}^{N_{\mathrm{S}}}\right\rangle
$$

Theorem 7.6. For each pair $N=\left(N_{\mathrm{F}}, N_{\mathrm{S}}\right)$ there exists $h_{N}$ such that

$$
\lim _{N_{\mathrm{F}}, N_{\mathrm{S}} \rightarrow \infty} \epsilon_{h_{N}}^{* N}=0 .
$$




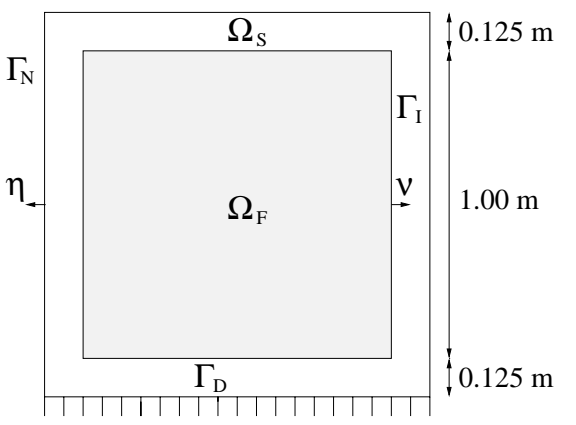

FIGURE 2. Steel cavity with water inside.

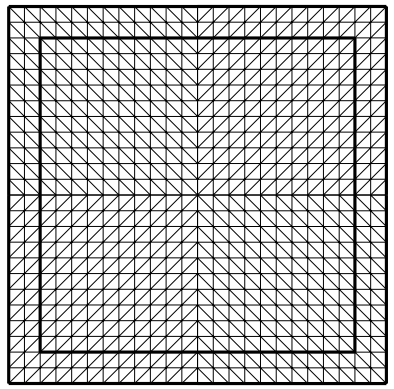

Figure 3. Meshes.

Proof. Since in $\mathbf{V}_{h}^{N, 1}$ there are not static liftings in the fluid, by reasoning as in the previous lemmas we have,

$$
\begin{aligned}
\epsilon_{h}^{* N}(\lambda) \leq & C\left(\left(\lambda_{N_{\mathrm{F}}+1}^{\mathrm{F}}\right)^{-\frac{\hat{r}}{2}}\left(\sum_{i=N_{\mathrm{F}}+1}^{\infty} \alpha_{i}^{2}\left(\lambda_{i}^{\mathrm{F}}\right)^{1+\hat{r}}\right)^{\frac{1}{2}}+\left(\lambda_{N_{\mathrm{S}}+1}^{\mathrm{S}}\right)^{-\frac{\hat{s}}{2}}\left(\sum_{m=N_{\mathrm{S}}+1}^{\infty} \beta_{m}^{2}\left(\lambda_{m}^{\mathrm{S}}\right)^{1+\hat{s}}\right)^{\frac{1}{2}}\right) \\
& +C h^{t}\left(\left(\lambda_{N_{\mathrm{F}}}^{\mathrm{F}}\right)^{\frac{1-\hat{r}}{2}} \sqrt{N_{\mathrm{F}}}+\left(\lambda_{N_{\mathrm{S}}}^{\mathrm{S}}\right)^{\frac{1-\hat{s}}{2}} \sqrt{N_{\mathrm{S}}}\right),
\end{aligned}
$$

with $\hat{r}<\frac{1}{2}$ and $\hat{s}$ the constant in Lemma 7.2. The theorem follows immediately.

\section{Numerical Results}

In this section we show some numerical results obtained by solving the discrete modal synthesis problem $\mathbf{V P}_{h}^{N}$ with a MATLAB code. We use the eigensolver eigs (based on Arnoldi iterations) for solving the involved spectral problems. We compare the computed eigenvalues with those obtained by a direct discretization with Lagrangian elements of the pressure/displacement formulation for the elastoacoustic problem (see [2]).

We consider the 2D fluid-solid coupled system described in Figure 2 and take the following physical parameters for steel and water as solid and fluid, respectively,

- $\nu=0.35$,

- $E=1.44 \times 10^{11} \mathrm{~Pa}$,

- $\rho_{\mathrm{S}}=7700 \mathrm{~kg} / \mathrm{m}^{3}$,

- $\rho_{\mathrm{F}}=1000 \mathrm{~kg} / \mathrm{m}^{3}$,

- $c=1430 \mathrm{~m} / \mathrm{s}$.

For a first test, we have used the meshes in Figure 3 and several values of $N_{\mathrm{F}}$ and $N_{\mathrm{S}}$ ranging between 5 and 50. We compare the obtained results with the corresponding discrete eigenvalues calculated without modal synthesis in [2]. In Figure 4 we show the relative error curves (in \%) $\frac{100\left|\lambda_{p h}^{N}-\lambda_{p h}\right|}{\left|\lambda_{p h}\right|}, p=1, \ldots, 4$, where $\lambda_{p h}^{N}$ is the $p$ th eigenvalue of problem $\mathbf{V P}_{h}^{N}$, and $\lambda_{p h}$ is the $p$ th eigenvalue of the method in [2] for the same meshes.

It is important to remark that, even for the lowest values of $N_{\mathrm{F}}$ and $N_{\mathrm{S}}$ that we have considered, the relative errors are very small, although they increase with $p$. For instance, for $p=4$, the relative error is around $1 \%$ when $N_{\mathrm{F}}=N_{\mathrm{S}}=5$, whereas for $N_{\mathrm{F}}=N_{\mathrm{S}}=10$ the error is around $0.1 \%$. On the other hand, it can also be seen from Figure 4 that the convergence rate is very small. Indeed, the error goes to zero as $N_{\mathrm{F}}, N_{\mathrm{S}} \rightarrow \infty$, but very slowly. Then, it is not worth to use a large number of uncoupled frequencies.

In practice, to approximate the lowest eigenfrequencies of the elastoacoustic coupled problem, it should be enough to consider a low number of uncoupled eigenfunctions on each media and solve $\mathbf{V P}_{h}^{N}$ with $h \rightarrow 0$. This is what we have done for the second test. We have fixed $N_{\mathrm{F}}=N_{\mathrm{S}}=10$ (we emphasize that, in this case, the 


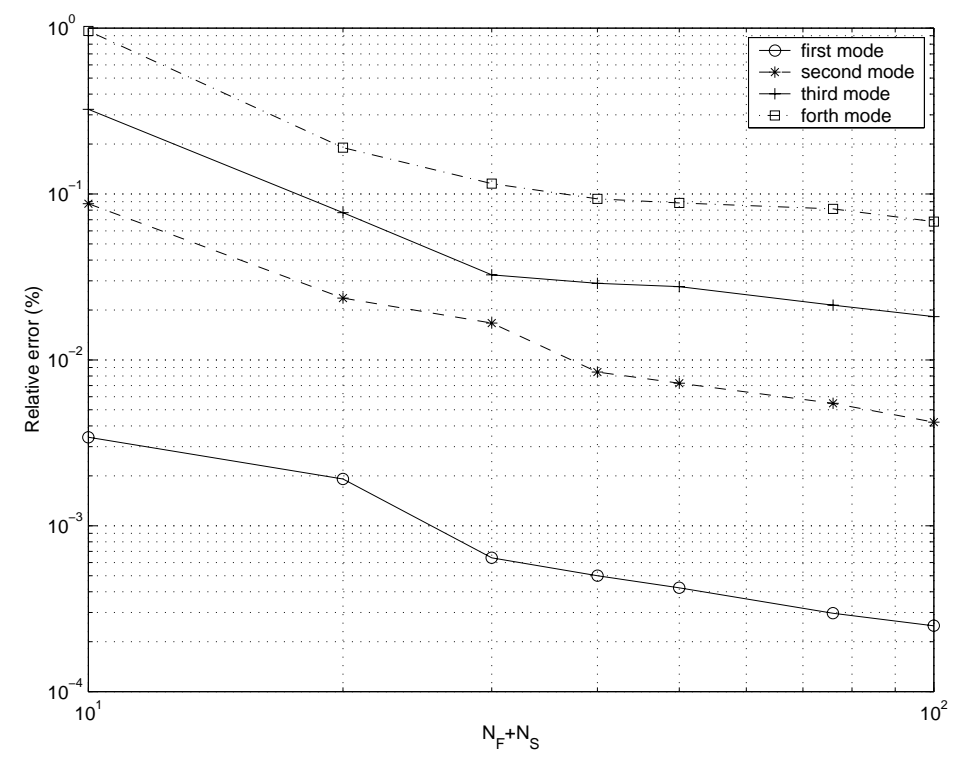

Figure 4. Error curves for a fixed mesh and varying values of $N_{\mathrm{F}}+N_{\mathrm{S}}$ (log-log scale).

matrix of the coupled problem (5.3) has only dimension 20) and used successive refinements of the mesh in Figure 3. Figure 5 shows the curves of the relative errors for the first 4 coupled modes, $\lambda_{p h}^{N}, p=1, \ldots, 4$, versus the number of degrees of freedom (d.o.f.) of the meshes. We have used as "exact" values those extrapolated from these results.

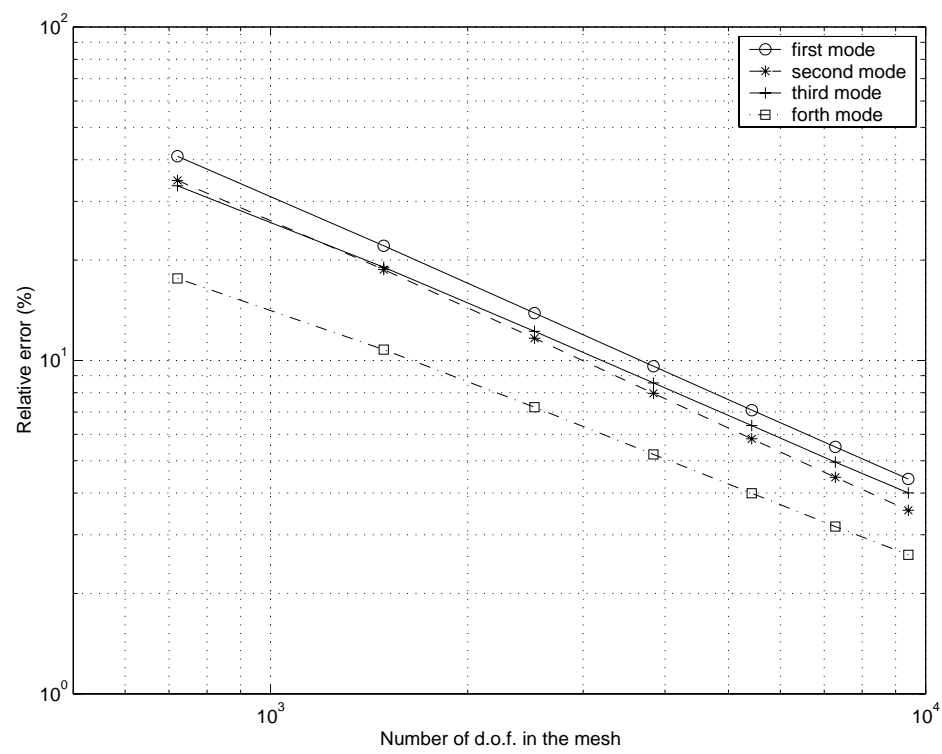

FiguRE 5. Error curves for fixed $N_{\mathrm{F}}=N_{\mathrm{S}}=10$ and successively refined meshes (log-log scale). 
TABLE 1. Extrapolated values with and without modal synthesis (MS).

\begin{tabular}{|c|c|c|c|}
\hline & Extrapolated value with MS & Extrapolated value without MS & Relative difference \\
\hline$\lambda_{1}$ & $391.796 \mathrm{~Hz}$ & $391.790 \mathrm{~Hz}$ & $0.0015 \%$ \\
$\lambda_{2}$ & $1399.318 \mathrm{~Hz}$ & $1399.350 \mathrm{~Hz}$ & $0.0023 \%$ \\
$\lambda_{3}$ & $2262.095 \mathrm{~Hz}$ & $2259.861 \mathrm{~Hz}$ & $0.0989 \%$ \\
$\lambda_{4}$ & $3206.232 \mathrm{~Hz}$ & $3202.063 \mathrm{~Hz}$ & $0.1302 \%$ \\
\hline
\end{tabular}

Let us remark that these extrapolated values are very close to the ones obtained with the method in [2], as it can be seen in Table 1. This means that, in practice, the results obtained with the method in [2] (without modal synthesis) and the results obtained with the modal synthesis method analyzed in this paper with fixed low values of $N_{\mathrm{F}}$ and $N_{\mathrm{S}}$, are almost the same for the first coupled modes. Nevertheless, the method with modal synthesis presents significant advantages since it does not require to solve high-dimensional coupled problems.

\section{REFERENCES}

[1] I. Babuška and J. Osborn, Eigenvalue problems. In Handbook of Numerical Analysis, Vol. II, P.G. Ciarlet and J.L. Lions, Eds., North Holland, Amsterdam (1991).

[2] A. Bermúdez, P. Gamallo, L. Hervella-Nieto and R. Rodríguez, Finite element analysis of the elastoacoustic problem using the pressure in the fluid. Preprint DIM 2001-05, Universidad de Concepción, Concepción, Chile (submitted).

[3] F. Bourquin, Analysis and comparison of several component mode synthesis methods on one-dimensional domains. Numer. Math. 58 (1990) 11-34.

[4] F. Bourquin, Component mode synthesis and eigenvales of second order operators: Discretization and algorithm. RAIRO Modél. Math. Anal. Numér. 26 (1992) 385-423.

[5] F. Bourquin, A pure displacement dynamic substructuring method with accurate pressure for elastoacoustics. Laboratoire Central des Ponts et Chaussées, R/94/05/7 (1994).

[6] P.G. Ciarlet, The Finite Element Method for Elliptic Problems. North-Holland, Amsterdam (1978).

[7] R. Craig and M.C.C. Bampton, Coupling of substructures for dynamic analysis. AIAA J. 6 (1968) 1313-1321.

[8] R.L. Goldman, Vibration analysis of dynamic analysis. AIAA J. 7 (1969) 1152-1154.

[9] P. Grisvard, Elliptic Problems in Nonsmooth Domains. Pitman, Boston (1985).

[10] P. Grisvard, Caractérisation de quelques espaces d'interpolation. Arch. Rat. Mech. Anal. 25 (1967) 40-63.

[11] L. Hervella-Nieto, Métodos de elementos finitos y reducción modal para problemas de interacción fluido-estructura. Ph.D. thesis, Publicaciones del Departamento de Matemática Aplicada, 27, Universidad de Santiago de Compostela (2000).

[12] W.C. Hurty, Dynamic analysis of structural systems using component modes. AIAA J. 4 (1965) 678-685.

[13] W.G. Kolata, Approximation in variationally posed eigenvalues problems. Numer. Math. 29 (1978) 159-171.

[14] J.L. Lions, Théorèmes de trace et d'interpolation (I). Ann. Scuola Norm. Sup. Pisa 13 (1959) 389-403.

[15] H.J.-P. Morand and R. Ohayon, Interactions Fluides-Structure. Masson, Paris (1996).

[16] H.J.-P. Morand and R. Ohayon, Substructure variational analysis of the vibration of coupled fluid-structure systems. Finite element results. Internat. J. Numer. Methods Engrg. 14 (1979) 741-755.

[17] J. Nečas, Les Méthodes Directes en Théorie des Équations Elliptiques. Masson, Paris (1967).

[18] G. Sandberg, A new strategy for solving fluid-structure problems. Internat. J. Numer. Methods Engrg. 38 (1995) 357-370.

[19] J. Wandinger, Analysis of small vibrations of coupled fluid-structure systems. Z. Angew. Math. Mech. 74 (1994) 37-42.

[20] J.-L. Zolesio, Interpolation d'espaces de Sobolev avec conditions aux limites de type mêlé. C. R. Acad. Sci. Paris Série A 285 (1982) 621-624. 